\title{
An existence result for multidimensional immiscible two-phase flows with discontinuous capillary pressure field*
}

\author{
Clément Cances ${ }^{\dagger} \quad$ Michel Pierre ${ }^{\ddagger}$
}

November 24, 2011

\begin{abstract}
We consider the system of equations governing an incompressible immiscible two-phase flow within an heterogeneous porous medium made of two different rock types. Since the capillary pressure function depends on the rock type, the capillary pressure field might be discontinuous at the interface between the rocks. We introduce multivalued phase pressures to give a sense to the transmission conditions at the interface. We prove the existence of a solution for such a flow by passing to the limit in regularizations of the problem.
\end{abstract}

keywords: two-phase flows, porous media, discontinuous capillarity, multivalued pressures

AMS subject classification: 35M33, 35Q86, 35K65, 76S05, 76T99

\section{Introduction}

The models of immiscible two-phase flows in porous media are often used to give a prediction of the motions of complex flows in subsoil, particularly in the frame of oil-engineering. So they have been widely studied, both from theoretical and numerical points of view. One of the main difficulty appearing in their study is linked to the degeneracy of the problem where one of the two phases vanishes.

Because of variations of the rock type, one has to take into account strong heterogeneities of the subsoil with respect to space in the model and to assume that the physical properties of the porous medium are even discontinuous in the case of severe variations of the rock type. It is well known that such discontinuities of the medium induce discontinuities of the fluid composition, but also discontinuous pressure fields (see [vDMdN95], [EEN98], [BDPvD03], [EEM06], [CGP09], [BLS09], [Can09]). While some mathematical analysis in the one-dimensional case has been carried out in [BDPvD03], [BLS09], [CGP09] and [Can09], ensuring the well-posedness of the problem, there is no existence result available for the solution of immiscible two-phase flows with discontinuous pressure fields in several dimensions, unless some strong assumptions are made in order to reduce the problem (see [CGP09], [EEM06]). In this paper, we propose to establish an existence result for the solution of the system of equations governing such a flow.

*Part of this work was supported by GNR MoMaS

${ }^{\dagger}$ UPMC Univ Paris 06, UMR 7598, Laboratoire Jacques-Louis Lions, F-75005, Paris (cances@ann.jussieu.fr)

${ }^{\ddagger}$ ENS Cachan Bretagne, UEB, IRMAR, (michel.pierre@bretagne.ens-cachan.fr) 


\subsection{Presentation of the problem}

For the sake of simplicity, we suppose that the porous medium, represented by a bounded open subset $\Omega$ with Lipschitz continuous boundary of $\mathbb{R}^{d}(d=2$ or 3$)$, is built of two homogeneous subdomains, represented by bounded open subsets $\left(\Omega_{i}\right)_{i \in\{1,2\}}$ with Lipschitz continuous boundaries such that $\Omega_{1} \cap \Omega_{2}=\emptyset$, and $\overline{\Omega_{1} \cup \Omega_{2}}=\bar{\Omega}$. We denote by $\Gamma \subset \Omega$ the interface between the two subdomains : $\bar{\Gamma}=\overline{\Omega_{1}} \cap \overline{\Omega_{2}}$. The extension of our paper to the case of a finite number of homogeneous

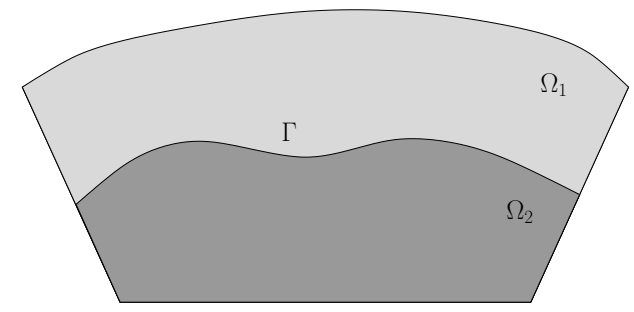

Figure 1: A example of domain $\Omega$ made of subdomains $\Omega_{1}$ and $\Omega_{2}$ separated by the interface $\Gamma$

subdomains $\Omega_{i}$ is straightforward as soon as each $\partial \Omega_{i}$ is Lipschitz continuous.

The porous medium $\Omega$ is supposed to be saturated by a moisture made of only two immiscible phases, the oil phase (for which the subscript $o$ stands) and the water phase (for which the subscript $w$ stands). One denotes by $s$ the oil saturation, and $(1-s)$ is thus the water saturation.

The motion of each phase in $\Omega_{i}$ is governed by the diphasic Darcy-Muskat laws (see e.g. [AS79]):

$$
\begin{aligned}
\phi_{i} \partial_{t} s-\operatorname{div}\left(\mathbf{K}_{i} \frac{k_{o, i}(s)}{\mu_{o}}\left(\nabla p_{o}-\rho_{o} \mathbf{g}\right)\right) & =0, \\
-\phi_{i} \partial_{t} s-\operatorname{div}\left(\mathbf{K}_{i} \frac{k_{w, i}(s)}{\mu_{w}}\left(\nabla p_{w}-\rho_{w} \mathbf{g}\right)\right) & =0,
\end{aligned}
$$

where $\phi_{i} \in(0,1)$ is the porosity of $\Omega_{i}$, the symmetric definite positive matrix $\mathbf{K}_{i}$ is the permeability of the rock $\Omega_{i}, k_{\alpha, i}$ is the relative permeability of the phase $\alpha \in\{o, w\}$ in $\Omega_{i}, \mu_{\alpha}>0$ is its viscosity, $p_{\alpha}$ its pressure, $\rho_{\alpha}$ its density and $\mathbf{g}$ stands for the gravity. In order to simplify the problem, we suppose that there are no irreducible saturations. More precisely, we do the following assumptions on the functions $k_{\alpha, i}$ :

Assumption 1 For $i \in\{1,2\}$,

- $k_{o, i} \in \mathcal{C}^{1}$ is (strictly) increasing on $[0,1]$ with $k_{o, i}(0)=0$ and $k_{o, i}(1)=1$;

- $k_{w, i} \in \mathcal{C}^{1}$ is (strictly) decreasing on $[0,1]$ with $k_{w, i}(0)=1$ and $k_{w, i}(1)=0$.

The difference between the phase pressures, so called capillary pressure, is given by the following simplified law

$$
p_{o}-p_{w}=\pi_{i}(s)
$$

We do the following reasonable assumption on the capillary pressure functions.

Assumption 2 For $i \in\{1,2\}$, the function $\pi_{i}$ belongs to $\mathcal{C}^{1}((0,1) ; \mathbb{R}) \cap L^{1}((0,1) ; \mathbb{R})$, and are (strictly) increasing. 
Note that the functions $\pi_{i}$ are not supposed to be bounded near 0 and 1 , but, thanks to the monotonicity of $\pi_{i}$, we can define

$$
\overline{\mathbb{R}} \ni \pi_{i}(0)=\lim _{s \rightarrow 0^{+}} \pi_{i}(s), \quad \overline{\mathbb{R}} \ni \pi_{i}(1)=\lim _{s \rightarrow 1^{-}} \pi_{i}(s) .
$$

It has been stressed in [ALV84] (and will be detailed later) that the natural topology for the phase pressures $p_{o}, p_{w}$ in $\Omega_{i}$ is (formally) governed by the estimate

$$
\iint\left(\frac{k_{o, i}(s)}{\mu_{o}}\left(\nabla p_{o}\right)^{2}+\frac{k_{w, i}(s)}{\mu_{w}}\left(\nabla p_{w}\right)^{2}\right) d x d t \leq C\left(1+\sum_{i \in\{1,2\}}\left\|\pi_{i}\right\|_{L^{1}(0,1)}\right)<+\infty .
$$

In particular, assume that $s(x, t)=0$ for some $x \in \Omega_{i}$, then it follows from Assumption 1 that $k_{o, i}(s(x, t))=0$. As a consequence, the control of the left-hand side in (4) provides no information on $p_{o}(x, t)$. But because of the relation (3), then the oil-pressure $p_{o}(x, t)$ cannot exceed the threshold value $p_{w}(x, t)+\pi_{i}(0)$, otherwise the oil-phase should be present. Hence, $p_{o}(x, t)$ should be defined in a multivalued way, i.e.

$$
s(x, t)=0 \Leftrightarrow p_{o}(x, t)=\left[-\infty, p_{w}(x, t)+\pi_{i}(0)\right] .
$$

Similarly, one has

$$
s(x, t)=1 \Leftrightarrow p_{w}(x, t)=\left[-\infty, p_{o}(x, t)-\pi_{i}(1)\right] .
$$

We deduce from (5) and (6) that the capillary pressure function $\pi_{i}$ has to be extended into a monotone graph $\tilde{\pi}_{i}$, defined by

$$
\tilde{\pi}_{i}(s)= \begin{cases}\pi_{i}(s) & \text { if } s \in(0,1) \\ {\left[-\infty, \pi_{i}(0)\right]} & \text { if } s=0 \\ {\left[\pi_{i}(1),+\infty\right]} & \text { if } s=1\end{cases}
$$

The capillary pressure graph $\tilde{\pi}_{i}$ admits a continuous inverse, denoted by $\pi_{i}^{-1}$, mapping $\overline{\mathbb{R}}$ to $[0,1]$, that, thanks to Assumption 2, satisfies

$$
\left\|\pi_{i}^{-1}\right\|_{L^{1}\left(\mathbb{R}_{-}\right)}+\left\|\pi_{i}^{-1}-1\right\|_{L^{1}\left(\mathbb{R}_{+}\right)}=\left\|\pi_{i}\right\|_{L^{1}((0,1))}<\infty .
$$

Remark 1. The most classical choices for the capillary pressure functions are the so-called Van Genuchten and Brooks-Corey capillary pressure functions, respectively defined by (we suppose here that the water phase is the wetting phase)

$$
\pi_{V G}(s)=A\left((1-s)^{-\frac{\nu}{\nu-1}}-1\right)^{\frac{1}{\nu}}, \quad \pi_{B C}(s)=B+C(1-s)^{-\frac{1}{\lambda}},
$$

where $A>0, B \geq 0, C>0, \nu>2$, and $\lambda>1$ are parameters depending on the rock type. These choices of capillary pressure functions satisfy Assumption 2, but are not bounded in the vicinity of 1. Therefore, allowing unbounded capillary pressures is of great interest in the current study. The boundedness of the energy (4) of the system only requires the capillary pressure functions to belong to $L^{1}(0,1)$, as prescribed by Assumption 2 .

Let us now focus on the transmission conditions at the interface $\Gamma$. On one hand, because of mass balance of each phase, both phase fluxes have to be continuous, i.e. for $\alpha \in\{o, w\}$, one has

$$
\sum_{i \in\{1,2\}}\left(\mathbf{K}_{i} \frac{k_{\alpha, i}(s)}{\mu_{\alpha}}\left(\nabla p_{\alpha}-\rho_{\alpha} \mathbf{g}\right)\right) \cdot \mathbf{n}_{i}=0 \quad \text { on } \Gamma
$$


where $\mathbf{n}_{i}$ denotes the outward normal to $\partial \Omega_{i}$. On the other hand, following [EEM06], we prescribe the continuity of the pressure of the mobile phases :

$$
k_{\alpha, 1}\left(s_{1}\right)\left(p_{\alpha, 1}-p_{\alpha, 2}\right)^{+}-k_{\alpha, 2}\left(s_{2}\right)\left(p_{\alpha, 2}-p_{\alpha, 1}\right)^{+}=0 \quad \text { on } \Gamma,
$$

where $s_{i}, p_{\alpha, i}$ denote the traces on $\Gamma$ from $\Omega_{i}$ of $s, p_{\alpha}$ respectively. The relation (9) claims that either the pressure of the phase $\alpha$ is continuous through the interface $\Gamma$, or the phase $\alpha$ is missing at the side of the interface where its pressure is larger. Using the multivalued formalism introduced in (5) and (6), the relation (9) is equivalent to

$$
p_{\alpha, 1} \cap p_{\alpha, 2} \neq \emptyset \quad \text { on } \Gamma \times(0, T) .
$$

We make more comments on these conditions in Remark 3.

In order to close the system, we impose a no-flux boundary condition for each phase on $\partial \Omega$

$$
\left(\mathbf{K}_{i} \frac{k_{\alpha, i}(s)}{\mu_{\alpha}}\left(\nabla p_{\alpha}-\rho_{\alpha} \mathbf{g}\right)\right) \cdot \mathbf{n}=0,
$$

where $\mathbf{n}$ denote the outward normal to $\Omega$, and an initial condition

$$
s_{0}(x) \in L^{\infty}(\Omega,[0,1]) .
$$

It is worth noticing that due to the choice of the boundary condition (11), the pressures are only determined up to an additive constant.

The purpose of this paper is to show that, after suitable reformulation, the problem (1)(3),(8),(10)-(12) admits a solution.

\subsection{Reformulation of the problem}

In order to avoid some of the difficulties linked to the degeneracies of the problem (1),(2), we follow the classical idea of introducing the so-called global pressure $P$ and Kirchhoff transform $\varphi_{i}(s)$ (see e.g. [CJ86], [AKM90], [Arb92]), and rewrite the equations (1)-(3) under the form

$$
\begin{aligned}
\phi_{i} \partial_{t} s-\operatorname{div}\left(\mathbf{K}_{i}\left(\frac{k_{o, i}(s)}{\mu_{o}}\left(\nabla P-\rho_{o} \mathbf{g}\right)+\nabla \varphi_{i}(s)\right)\right) & =0 \\
-\operatorname{div}\left(\mathbf{K}_{i}\left(M_{i}(s) \nabla P-\zeta_{i}(s) \mathbf{g}\right)\right) & =0
\end{aligned}
$$

with

$$
\begin{gathered}
M_{i}(s)=\frac{k_{o, i}(s)}{\mu_{o}}+\frac{k_{w, i}(s)}{\mu_{w}}, \quad \varphi_{i}(s)=\int_{0}^{s} \frac{k_{o, i}(a) k_{w, i}(a)}{k_{o, i}(a) \mu_{w}+k_{w, i}(a) \mu_{o}} \pi_{i}^{\prime}(a) d a, \\
\zeta_{i}(s)=\frac{k_{o, i}(s)}{\mu_{o}} \rho_{o}+\frac{k_{w, i}(s)}{\mu_{w}} \rho_{w}
\end{gathered}
$$

and, for any $\pi \in \tilde{\pi}_{i}(s)$,

$$
P=p_{w}+\lambda_{w, i}(\pi)=p_{o}+\lambda_{o, i}(\pi)
$$


where, for all $\pi \in \mathbb{R}$, we have set

$$
\begin{aligned}
\lambda_{w, i}(\pi) & =\int_{0}^{\pi} \frac{k_{o, i}\left(\pi_{i}^{-1}(a)\right)}{k_{o, i}\left(\pi_{i}^{-1}(a)\right)+\frac{\mu_{o}}{\mu_{w}} k_{w, i}\left(\pi_{i}^{-1}(a)\right)} d a, \\
\lambda_{o, i}(\pi) & =-\int_{0}^{\pi} \frac{k_{w, i}\left(\pi_{i}^{-1}(a)\right)}{\frac{\mu_{w}}{\mu_{o}} k_{o, i}\left(\pi_{i}^{-1}(a)\right)+k_{w, i}\left(\pi_{i}^{-1}(a)\right)} d a .
\end{aligned}
$$

Firstly, $P$ is always single-valued, and does not depend on the choice of $\pi \in \tilde{\pi}_{i}(s)$. Indeed, if $s \in(0,1)$, both expressions of $P$ are single-valued. If $s=0$, then $p_{w}$ is single-valued, and, for all $\pi \leq \pi_{i}(0)$,

$$
\int_{\pi_{i}(0)}^{\pi} \frac{k_{o, i}\left(\pi_{i}^{-1}(a)\right)}{k_{o, i}\left(\pi_{i}^{-1}(a)\right)+\frac{\mu_{o}}{\mu_{w}} k_{w, i}\left(\pi_{i}^{-1}(a)\right)} d a=0,
$$

ensuring that $P=p_{w}+\lambda_{w, i}(\pi)$ is single-valued. In the case $s=1$, one has to use the relation $P=p_{o}+\lambda_{o, i}(\pi)$. Notice that for all $\pi \in \mathbb{R}$, one has

$$
\lambda_{w, i}(\pi)-\lambda_{o, i}(\pi)=\pi
$$

Note also that

$$
M_{i}(s) \nabla P=\frac{k_{o, i}(s)}{\mu_{o}} \nabla p_{o}+\frac{k_{w, i}(s)}{\mu_{w}} \nabla p_{w} .
$$

The function $\varphi_{i}^{\prime}$ is given by

$$
\varphi_{i}^{\prime}(s)=\frac{k_{o, i}(s) k_{w, i}(s)}{k_{o, i}(s) \mu_{w}+k_{w, i}(s) \mu_{o}} \pi_{i}^{\prime}(s)
$$

where $\pi_{i}^{\prime}(s)$ can possibly tend to $+\infty$ as $s$ tends to 0 or 1 , but simultaneously, the ratio $\frac{k_{o, i}(s) k_{w, i}(s)}{k_{o, i}(s) \mu_{w}+k_{w, i}(s) \mu_{o}}$ tends to 0 . By the following assumption, we assume that the product remains bounded.

Assumption 3 The functions $\varphi_{i}$ are Lipschitz continuous on $[0,1]$.

Thanks to Assumption 1 and since $\pi_{i}$ is supposed to be increasing, the functions $\varphi_{i}$ defined above are such that $\varphi_{i}^{-1}$ are continuous functions on $\left[\varphi_{i}(0), \varphi_{i}(1)\right]$. We define the quantity

$$
\alpha_{M}=\min _{i \in\{1,2\}}\left(\min _{s \in[0,1]} M_{i}(s)\right)
$$

it is then easy to check that $\alpha_{M}>0$.

We now focus on the transmission conditions. The conservation of the oil-phase at the interface can be written

$$
\sum_{i \in\{1,2\}} \mathbf{K}_{i}\left(\frac{k_{o, i}(s)}{\mu_{o}}\left(\nabla P-\rho_{o} \mathbf{g}\right)+\nabla \varphi_{i}(s)\right) \cdot \mathbf{n}_{i}=0 \quad \text { on } \Gamma,
$$

while the conservation of the total flux at the interface yields

$$
\sum_{i \in\{1,2\}}\left(\mathbf{K}_{i}\left(M_{i}(s) \nabla P-\zeta_{i}(s) \mathbf{g}\right)\right) \cdot \mathbf{n}_{i}=0 \quad \text { on } \Gamma .
$$


The relation (10) on the interface $\Gamma$ implies that

$$
\text { there exists } \pi \in \tilde{\pi}_{1}\left(s_{1}\right) \cap \tilde{\pi}_{2}\left(s_{2}\right) \text { s.t. } P_{1}-\lambda_{w, 1}(\pi)=P_{2}-\lambda_{w, 2}(\pi) .
$$

Remark 2. In (22), requiring that $P_{1}-\lambda_{w, 1}(\pi)=P_{2}-\lambda_{w, 2}(\pi)$ corresponds to imposing the continuity (in the multivalued sense (10)) of the water pressure. By adding $\pi$ to this relation, we obtain, thanks to (18), that

$$
P_{1}-\lambda_{w, 1}(\pi)+\pi=P_{1}-\lambda_{o, 1}(\pi)=P_{2}-\lambda_{w, 2}(\pi)+\pi=P_{2}-\lambda_{o, 2}(\pi) .
$$

Then we also recover the continuity of the oil-pressure (in the same weak sense (10)), so that the relation (22) contains the continuity of both pressures.

Concerning the boundary conditions, the no-flux boundary condition for each phase is replaced by

$$
\begin{array}{rc}
\mathbf{K}_{i}\left(\frac{k_{o, i}(s)}{\mu_{o}}\left(\nabla P-\rho_{o} \mathbf{g}\right)+\nabla \varphi_{i}(s)\right) \cdot \mathbf{n}=0 & \text { on } \partial \Omega \cap \partial \Omega_{i}, \\
\left(\mathbf{K}_{i}\left(M_{i}(s) \nabla P-\zeta_{i}(s) \mathbf{g}\right)\right) \cdot \mathbf{n}=0 & \text { on } \partial \Omega \cap \partial \Omega_{i} .
\end{array}
$$

For the sake of simplicity, we choose to consider a finite time horizon $T>0$, then we denote by $Q_{T}$ (resp. $\left.Q_{i, T}\right)$ the cylinder $\Omega \times(0, T)\left(\operatorname{resp} \Omega_{i} \times(0, T)\right)$. All along the paper, for any $f \in$ $\{\phi, \mathbf{K}, \ldots\}$, we denote by $x \mapsto f(s, x)$ the piecewise constant function equal to $f_{i}(s)$ if $x \in \Omega_{i}$.

Definition 1.1 (weak solution) A couple $(s, P)$ is said to be a weak solution to the problem (12)(14), (20)-(24) in the cylinder $Q_{T}$ if it fulfills the following points:

1. $s \in L^{\infty}\left(Q_{T},[0,1]\right), \phi \partial_{t} s \in L^{2}\left((0, T) ;\left(H^{1}(\Omega)\right)^{\prime}\right)$ and $\varphi_{i}(s) \in L^{2}\left((0, T) ; H^{1}\left(\Omega_{i}\right)\right)$;

2. $P \in L^{2}\left((0, T) ; H^{1}\left(\Omega_{i}\right)\right)$ for $i \in\{1,2\}$ and $\int_{\Omega} P(x, t) d x=0$ for a.e. $t \in(0, T)$;

3. there exists a measurable function $\pi$ mapping $\Gamma \times(0, T)$ to $\overline{\mathbb{R}}$ such that, for almost all $(x, t) \in$ $\Gamma \times(0, T)$,

$$
\pi \in \tilde{\pi}_{1}\left(s_{1}\right) \cap \tilde{\pi}_{2}\left(s_{2}\right) \text { and } P_{1}-\lambda_{w, 1}(\pi)=P_{2}-\lambda_{w, 2}(\pi) ;
$$

4. for all $\psi \in L^{2}\left((0, T) ; H^{1}(\Omega)\right)$, one has

$$
\begin{aligned}
& \iint_{Q_{T}} \phi s \partial_{t} \psi d x d t+\int_{\Omega} \phi s_{0} \psi(\cdot, 0) d x \\
& \quad+\sum_{i \in\{1,2\}} \iint_{Q_{i, T}} \mathbf{K}_{i}\left(\frac{k_{o, i}(s)}{\mu_{o}}\left(\nabla P-\rho_{o} \mathbf{g}\right)+\nabla \varphi_{i}(s)\right) \cdot \nabla \psi d x d t=0 ;
\end{aligned}
$$

5. for all $\psi \in L^{2}\left((0, T) ; H^{1}(\Omega)\right)$, one has

$$
\sum_{i \in\{1,2\}} \iint_{Q_{i, T}}\left(\mathbf{K}_{i}\left(M_{i}(s) \nabla P-\zeta_{i}(s) \mathbf{g}\right)\right) \cdot \nabla \psi d x d t=0 .
$$


Remark 3. In the one-dimensional case, the problem reduces to a single degenerate parabolic equation in each subdomain $\Omega_{i}$. Formally, this equation can be written with the extended capillary pressure $\pi: \Omega_{i} \rightarrow \overline{\mathbb{R}}$ as main unknown, i.e.

$$
\phi_{i} \partial_{t} \pi_{i}^{-1}(\pi)+\partial_{x}\left(q f_{i}(\pi)+\gamma_{i}(\pi) g-\lambda_{i}(\pi) \partial_{x} \pi\right)=0 \quad \text { in } Q_{i, T}
$$

for some functions $f_{i}, \gamma_{i}$ and $\lambda_{i}$. Prescribing the continuity of $\pi$ at the interface turns out to be sufficient to obtain an existence/uniqueness result on the saturation $s:=\pi_{i}^{-1}(\pi)$, thanks to a $L^{1}$ contraction principle established in [Can09] (see also [CGP09]). The traces $s_{i}$ of $s$ then naturally satisfy

$$
\pi \in \tilde{\pi}_{1}\left(s_{1}\right) \cap \tilde{\pi}_{2}\left(s_{2}\right) \neq \emptyset \quad \text { on } \Gamma \times(0, T) .
$$

In the multidimensional case, the problem in each $\Omega_{i}$ really consists in two equations (1),(2) on $p_{o}, p_{w}$. Therefore, prescribing the single relation (27) is clearly not sufficient for obtaining a uniqueness frame for $(s, P)$. Indeed, if $(s, P)$ is a weak solution of the problem with the single continuity condition (27) instead of (22), i.e. if it satisfies the points 1,2,4 and 5 of Definition 1.1 and the continuity of the capillary pressure (27), then, for any function $\kappa \in L^{2}(0, T)$, defining

$$
\tilde{P}_{\kappa}(x, t)=P(x, t)+\kappa(t)\left(\left|\Omega_{2}\right| \mathbf{1}_{\Omega_{1}}(x)-\left|\Omega_{1}\right| \mathbf{1}_{\Omega_{2}}(x)\right),
$$

the couple $\left(s, \tilde{P}_{\kappa}\right)$ also satisfies the points $1,2,4$ and 5 of Definition 1.1 and the continuity of the capillary pressure (27). In order to eliminate this spurious degree of freedom, one has to fix the jump of the global pressure. The convenient way to do it consists in prescribing (in the graph sense prescribed in the paper) the continuity of the phase pressure $p_{w}$, i.e.

$$
P_{1}-\lambda_{w, 1}(\pi)=P_{2}-\lambda_{w, 2}(\pi) .
$$

Since the capillary pressure pressure $\pi$ is itself continuous, so does $p_{o}$ thanks to Remark 2 .

The paper is devoted to the proof of the following theorem.

Theorem 1 (main result) Under Assumptions 1,2 and 3, there exists a weak solution to the problem (12)-(14), (20)-(24) in the sense of Definition 1.1.

It is well known that for suitable initial and boundary conditions, the flow governed by the equations (13)-(14) admits a solution (see e.g. [ALV84], [AD85], [CJ86], [AKM90], [Arb92] or [Che01]) in the case where the physical characteristics of the domain do not depend on space, or at least sufficiently smoothly. In the case considered here, the difficulty will come from the fact that the physical properties of the medium $\Omega$-particularly the capillary pressure curve - are discontinuous with respect to space at the interface $\Gamma$. The effects of space depending capillarities have been widely studied during the last years. Analytical results have been provided by [ABE96], [vDMdN95], [BDPvD03], [Can08], [CGP09]. Effective models have been provided in [BH95a], [vDMP02], [vDEHP07] and [Sch08] using homogenization techniques. Some numerical schemes have been introduced [EEN98], [EMS09] and studied [EEM06], [Can09], [BCH]. It has been pointed out in [Can10a], [Can10b] and [Can10c] (see also [AC11]) that the orientation of the capillary forces at the interface has a strong influence on the qualitative behavior of the saturation profile. 


\subsection{Organization of the paper}

In $\S 2$, we introduce a simplified problem, where the pressure of both phases is (strongly) continuous at the interface. This can be done under a compatibility condition of the capillary forces, that is

$$
\pi_{1}(0)=\pi_{2}(0) \in \mathbb{R}, \quad \pi_{1}(1)=\pi_{2}(1) \in \mathbb{R} .
$$

If the functions $\pi_{i}$ satisfy the above condition, the capillarity curves are said to be matching. In that case, the existence of a weak solution is proven using a spatial regularization of the function $x \mapsto \pi(\cdot, x)$, i.e. by introducing a thin transition layer between the two rocks.

Section 3 is devoted to the end of the proof of Theorem 1. We will show that the problem with non-matching capillarity curves, i.e. when the condition (28) is not satisfied, can be approximated by problems with matching capillarity curves studied in $\S 2$. Compactness properties on the family

of approximate solutions will allow us to exhibit a weak solution in the sense of Definition 1.1 as a limit value.

\section{The problem with matching capillary pressure curves}

In this section, we assume that the capillary pressure functions $\pi_{i}$ belong to $\mathcal{C}^{1}([0,1] ; \mathbb{R})$, and fulfill the relation (28), so that the relation (22) turns to

$$
\pi_{1}\left(s_{1}\right)=\pi_{2}\left(s_{2}\right) \text { and } P_{1}-\lambda_{w, 1}\left(\pi_{1}\left(s_{1}\right)\right)=P_{2}-\lambda_{w, 2}\left(\pi_{2}\left(s_{2}\right)\right) .
$$

So the pressure of each phase is continuous at the interface $\Gamma$, i.e.

$$
p_{o, 1}=p_{o, 2}, \quad p_{w, 1}=p_{w, 2} .
$$

Theorem 2 Under assumption (28), there exists a weak solution $(s, P)$ to the problem (12)-(14), (20), (21), (23), (24), (29) in the sense of Definition 1.1.

Remark 4. The result stated in Theorem 2 is very close to the main result of the paper [BH95b]. However, it seems that there is a technical gap in the proof suggested in [BH95b] and detailed in [Hid93]. For this reason, we choose to give another proof of this theorem. But we stress the fact that the main result proposed in [BH95b] is true and that numerous ideas presented here have already been proposed in [BH95b], [Hid93]. In particular, the homogeneization result published in [BH95a] relies on correct preliminaries.

\subsection{The regularized problems}

Let $\epsilon>0$. In order to obtain regular phase pressures, the problem is regularized as follows: find $\left(s^{\epsilon}, p_{o}^{\epsilon}, p_{w}^{\epsilon}\right)$ such that

$$
\begin{array}{r}
\phi_{i} \partial_{t} s^{\epsilon}-\operatorname{div}\left(\mathbf{K}_{i} \frac{k_{o, i}\left(s^{\epsilon}\right)}{\mu_{o}}\left(\nabla p_{o}^{\epsilon}-\rho_{o} \mathbf{g}\right)\right)=\epsilon \Delta \pi_{i}\left(s^{\epsilon}\right) \text { in } Q_{i, T} \\
-\phi_{i} \partial_{t} s^{\epsilon}-\operatorname{div}\left(\mathbf{K}_{i} \frac{k_{w, i}\left(s^{\epsilon}\right)}{\mu_{w}}\left(\nabla p_{w}^{\epsilon}-\rho_{w} \mathbf{g}\right)\right)=-\epsilon \Delta \pi_{i}\left(s^{\epsilon}\right) \text { in } Q_{i, T}
\end{array}
$$

where $p_{o}^{\epsilon}-p_{w}^{\epsilon}=\pi_{i}\left(s^{\epsilon}\right)$. We require the continuity of the regularized pressures at the interface, i.e.

$$
p_{o, 1}^{\epsilon}=p_{o, 2}^{\epsilon}, \quad p_{w, 1}^{\epsilon}=p_{w, 2}^{\epsilon} \text { on } \Gamma \times(0, T),
$$


as well as the volume balances

$$
\begin{gathered}
\sum_{i \in\{1,2\}}\left(\mathbf{K}_{i} \frac{k_{o, i}\left(s^{\epsilon}\right)}{\mu_{o}}\left(\nabla p_{o}^{\epsilon}-\rho_{o} \mathbf{g}\right)+\epsilon \nabla \pi_{i}\left(s^{\epsilon}\right)\right) \cdot \mathbf{n}_{i}=0 \text { on } \Gamma \times(0, T), \\
\sum_{i \in\{1,2\}}\left(\mathbf{K}_{i} \frac{k_{w, i}\left(s^{\epsilon}\right)}{\mu_{w}}\left(\nabla p_{w}^{\epsilon}-\rho_{w} \mathbf{g}\right)-\epsilon \nabla \pi_{i}\left(s^{\epsilon}\right)\right) \cdot \mathbf{n}_{i}=0 \text { on } \Gamma \times(0, T) .
\end{gathered}
$$

No-flux boundary conditions are considered on $\partial \Omega \times(0, T)$, and we keep the initial data $s^{\epsilon}(\cdot, 0)=s_{0}$ in $\Omega$

In order to use an existing result (adaptation of Theorem 1 in [Arb92] or Theorem 2.1 in [Che01]), we introduce a smooth regularization of $\Omega$, consisting in introducing a thin transition layer to replace $\Gamma$. Let $\delta>0$, we define the Lipschitz continuous function $H^{\delta}$ on $\Omega$ by

$$
H^{\delta}(x)=\frac{1}{2}\left(1-\min \left(\frac{d\left(x, \Omega_{1}\right)}{\delta}, 1\right)+\min \left(\frac{d\left(x, \Omega_{2}\right)}{\delta}, 1\right)\right)
$$

so that $H^{\delta}(x)=1$ if $d\left(x, \Omega_{2}\right) \geq \delta$ and $H^{\delta}(x)=0$ if $d\left(x, \Omega_{1}\right) \geq \delta$. Let $f \in\left\{\mathbf{K}, \phi, k_{\alpha}, \pi\right\}$ piecewise constant on $\Omega$ with respect to space, we define the function

$$
f^{\delta}:(s, x) \mapsto f_{1}(s) H^{\delta}(x)+f_{2}(s)\left(1-H^{\delta}(x)\right)
$$

which has been built in order to be Lipschitz continuous with respect to the space variable $x$. For $g \in\left\{M, \varphi, \zeta, \lambda_{w}\right\}$, we denote by $g^{\delta}$ the function obtained by using $k_{\alpha}^{\delta}, \pi^{\delta}$ instead of $k_{\alpha}, \pi$ in the definition of $g$.

We define the fully regularized problem by: find $\left(s^{\epsilon, \delta}, p_{o}^{\epsilon, \delta}, p_{w}^{\epsilon, \delta}\right)$ such that

$$
\begin{array}{r}
\phi^{\delta} \partial_{t} s^{\epsilon, \delta}-\operatorname{div}\left(\mathbf{K}^{\delta} \frac{k_{o}^{\delta}\left(s^{\epsilon, \delta}\right)}{\mu_{o}}\left(\nabla p_{o}^{\epsilon, \delta}-\rho_{o} \mathbf{g}\right)\right)=\epsilon \Delta \pi^{\delta}\left(s^{\epsilon, \delta}\right) \text { in } Q_{T}, \\
-\phi^{\delta} \partial_{t} s^{\epsilon, \delta}-\operatorname{div}\left(\mathbf{K}^{\delta} \frac{k_{w}^{\delta}\left(s^{\epsilon, \delta}\right)}{\mu_{w}}\left(\nabla p_{w}^{\epsilon, \delta}-\rho_{w} \mathbf{g}\right)\right)=-\epsilon \Delta \pi^{\delta}\left(s^{\epsilon, \delta}\right) \text { in } Q_{T},
\end{array}
$$

where $p_{o}^{\epsilon, \delta}-p_{w}^{\epsilon, \delta}=\pi^{\delta}\left(s^{\epsilon, \delta}\right)$ in $Q_{T}$. Once again, we consider no-flux boundary conditions on $\partial \Omega \times$ $(0, T)$.

In the sequel, we denote by $m_{\mathbf{K}_{i}}$ (resp. $M_{\mathbf{K}_{i}}$ ) the smallest (resp. largest) eigenvalue of the symmetric definite positive matrix $\mathbf{K}_{i}$, and by $m_{\mathbf{K}}:=\min _{i} m_{\mathbf{K}_{i}}$ and $M_{\mathbf{K}}:=\max _{i} M_{\mathbf{K}_{i}}$.

Proposition 2.1 Under Assumption (28), there exist $s^{\epsilon, \delta} \in L^{\infty}\left(Q_{T},[0,1]\right) \cap \mathcal{C}\left([0, T] ; L^{2}(\Omega)\right)$ with $\partial_{t} s^{\epsilon, \delta} \in L^{2}\left(\left(0, T ; H^{-1}(\Omega)\right)\right.$, and $p_{o}^{\epsilon, \delta}, p_{w}^{\epsilon, \delta} \in L^{2}\left((0, T) ; H^{1}(\Omega)\right)$ (hence $\pi^{\delta}\left(s^{\epsilon, \delta}\right) \in L^{2}\left((0, T) ; H^{1}(\Omega)\right)$ ) solution to the system (32). Moreover, the following energy estimate holds: there exists $C$ depending only on $\phi, \mathbf{K}, \rho_{\alpha}, \mu_{\alpha}, \Omega, T, \mathbf{g}$ (but neither on $\epsilon$ nor on $\delta$ ) such that

$$
\begin{aligned}
& \frac{m_{\mathbf{K}}}{2} \sum_{\alpha \in\{o, w\}} \iint_{Q_{T}} \frac{k_{\alpha, i}\left(s^{\epsilon, \delta}\right)}{\mu_{\alpha}}\left(\nabla p_{\alpha}^{\epsilon, \delta}\right)^{2} d x d t \\
& \quad+\epsilon \iint_{Q_{T}}\left(\nabla \pi\left(s^{\epsilon, \delta}\right)\right)^{2} d x d t \leq C\left(1+\max _{i \in\{1,2\}}\left\|\pi_{i}\right\|_{L^{1}(0,1)}\right) .
\end{aligned}
$$


Denoting by

$$
P^{\epsilon, \delta}=p_{w}^{\epsilon, \delta}+\lambda_{w}^{\delta}\left(\pi^{\delta}\left(s^{\epsilon, \delta}\right)\right),
$$

one can furthermore require that

$$
\int_{\Omega} P^{\epsilon, \delta}(x, t) d x=0 \quad \text { for a.e. } t \in[0, T] .
$$

Proof. The existence proofs carried out in [Arb92] and [Che01], dealing with the case $\epsilon=0$, can be mimicked for $\epsilon>0$. This particularly yields the existence of a function $\pi^{\epsilon, \delta} \in L^{2}\left((0, T) ; H^{1}(\Omega)\right)$ and $p_{o}^{\epsilon, \delta}, p_{o}^{\epsilon, \delta} \in L^{2}\left((0, T) ; H^{1}(\Omega)\right)$ such that

$$
\begin{array}{r}
\phi^{\delta} \partial_{t}\left(\pi^{\delta}\right)^{-1}\left(\pi^{\epsilon, \delta}\right)-\operatorname{div}\left(\mathbf{K}^{\delta} \frac{k_{o}^{\delta}\left(\left(\pi^{\delta}\right)^{-1}\left(\pi^{\epsilon, \delta}\right)\right)}{\mu_{o}}\left(\nabla p_{o}^{\epsilon, \delta}-\rho_{o} \mathbf{g}\right)\right)=\epsilon \Delta \pi^{\epsilon, \delta}, \\
-\phi^{\delta} \partial_{t}\left(\pi^{\delta}\right)^{-1}\left(\pi^{\epsilon, \delta}\right)-\operatorname{div}\left(\mathbf{K}^{\delta} \frac{k_{w}^{\delta}\left(\left(\pi^{\delta}\right)^{-1}\left(\pi^{\epsilon, \delta}\right)\right)}{\mu_{w}}\left(\nabla p_{w}^{\epsilon, \delta}-\rho_{w} \mathbf{g}\right)\right)=-\epsilon \Delta \pi^{\epsilon, \delta},
\end{array}
$$

where $p_{o}^{\epsilon, \delta}-p_{w}^{\epsilon, \delta}=\pi^{\epsilon, \delta}$ and $\pi^{\epsilon, \delta}(\cdot, 0)=\left(\pi^{\delta}\right)^{-1}\left(s_{0}\right)$. Note that, thanks to classical arguments, the solution to the above system satisfies

$$
\pi^{\epsilon, \delta}(x, t) \in \mathcal{I}_{\pi}:=\left[\min _{j}\left(\pi_{j}(0)\right), \max _{j}\left(\pi_{j}(1)\right)\right]=\left[\pi^{\delta}(0, x), \pi^{\delta}(1, x)\right], \text { a.e. in } Q_{T} .
$$

It is important to remark that, thanks to Assumption (28), the interval $\mathcal{I}_{\pi}$ does not depend on $x$. Since the function $\pi^{\delta}(\cdot, x)$ is a homeomorphism from $[0,1]$ to $\mathcal{I}_{\pi}$, for a.e. $(x, t) \in Q_{T}$, there exists $s^{\epsilon, \delta}(x, t)$ such that $\pi^{\epsilon, \delta}(x, t)=\pi^{\delta}\left(s^{\epsilon, \delta}(x, t)\right)$. Thus we obtain the existence of a function $s^{\epsilon, \delta} \in L^{\infty}\left(Q_{T},[0,1]\right)$ and $p_{o}^{\epsilon, \delta}, p_{o}^{\epsilon, \delta} \in L^{2}\left((0, T) ; H^{1}(\Omega)\right)$ satisfying the system (32). The fact that $s^{\epsilon, \delta}$ belongs to $\mathcal{C}\left([0, T] ; L^{2}(\Omega)\right)$ can be deduced for example from the result of [CG11] applied on the first equation of $(32)$.

Choosing $p_{o}^{\epsilon, \delta}$ as test function in the first equation, $p_{w}^{\epsilon, \delta}$ in the second one and summing yields:

$$
\begin{aligned}
& \left\langle\phi^{\delta} \partial_{t} s^{\delta, \epsilon}, \pi^{\delta}\left(s^{\delta, \epsilon}\right)\right\rangle+\sum_{\alpha \in\{o, w\}} \iint_{Q_{T}}\left(\frac{k_{\alpha}^{\delta}\left(s^{\delta, \epsilon}\right)}{\mu_{\alpha}} \mathbf{K}^{\delta} \nabla p_{\alpha}^{\delta, \epsilon} \cdot \nabla p_{\alpha}^{\delta, \epsilon}\right) d x d t \\
& \quad+\epsilon \iint_{Q_{T}}\left|\nabla \pi^{\delta}\left(s^{\delta, \epsilon}\right)\right|^{2} d x d t-\sum_{\alpha \in\{o, w\}} \iint_{Q_{T}} \frac{k_{\alpha}^{\delta}\left(s^{\delta, \epsilon}\right)}{\mu_{\alpha}} \mathbf{K}^{\delta} \nabla p_{\alpha}^{\delta, \epsilon} \cdot \rho_{\alpha} \mathbf{g} d x d t=0 .
\end{aligned}
$$

Denoting by $\Pi^{\delta}(s, x)=\int_{0}^{s} \pi^{\delta}(a, x) d a$, it is classical (see e.g. Lemma 4 in [Car99]) that

$$
\begin{aligned}
& \left\langle\phi^{\delta} \partial_{t} s^{\delta, \epsilon}, \pi^{\delta}\left(s^{\delta, \epsilon}\right)\right\rangle=\int_{\Omega} \phi^{\delta}(x) \Pi^{\delta}\left(s^{\delta, \epsilon}\right)(x, T) d x-\int_{\Omega} \phi^{\delta}(x) \Pi^{\delta}\left(s_{0}\right)(x) d x \\
& \geq \quad-2 \int_{\Omega} \phi^{\delta}(x) \int_{0}^{1}\left|\pi^{\delta}(a, x)\right| d a d x \geq-2|\Omega|\left(\max _{i} \phi_{i}\right)\left(\max _{i}\left\|\pi_{i}\right\|_{L^{1}(0,1)}\right) .
\end{aligned}
$$


Since each $\mathbf{K}_{i}$ is a symmetric positive definite matrix, $\mathbf{K}^{\delta}(x)$ also for all $x \in \Omega$. We denote by $m_{\mathbf{K}^{\delta}}(x)$ (resp. $M_{\mathbf{K}^{\delta}}(x)$ ) its smaller (resp. larger) eigenvalue. Then it is easy to check that for all $x \in \Omega$, one has

$$
m_{\mathbf{K}}=\min _{i \in\{1,2\}} m_{\mathbf{K}_{i}} \leq m_{\mathbf{K}^{\delta}}(x), \quad M_{\mathbf{K}}=\max _{i \in\{1,2\}} M_{\mathbf{K}_{i}} \geq M_{\mathbf{K}^{\delta}}(x) .
$$

This provides that for $\alpha \in\{o, w\}$, one has

$$
\iint_{Q_{T}}\left(\frac{k_{\alpha}^{\delta}\left(s^{\delta, \epsilon}\right)}{\mu_{\alpha}} \mathbf{K}^{\delta} \nabla p_{\alpha}^{\delta, \epsilon} \cdot \nabla p_{\alpha}^{\delta, \epsilon}\right) d x d t \geq m_{\mathbf{K}} \iint_{Q_{T}} \frac{k_{\alpha}^{\delta}\left(s^{\delta, \epsilon}\right)}{\mu_{\alpha}}\left|\nabla p_{\alpha}^{\epsilon, \delta}\right|^{2} d x d t
$$

From Cauchy-Schwarz inequality, one has

$$
\begin{aligned}
& \iint_{Q_{T}} \frac{k_{\alpha}^{\delta}\left(s^{\delta, \epsilon}\right)}{\mu_{\alpha}} \mathbf{K}^{\delta} \nabla p_{\alpha}^{\delta, \epsilon} \cdot \rho_{\alpha} \mathbf{g} d x d t \\
& \quad \leq \quad M_{\mathbf{K}} \frac{\rho_{\alpha}}{\sqrt{\mu_{\alpha}}}|\mathbf{g}|\left|Q_{T}\right|^{\frac{1}{2}}\left(\iint_{Q_{T}} \frac{k_{\alpha}^{\delta}\left(s^{\delta, \epsilon}\right)}{\mu_{\alpha}}\left|\nabla p_{\alpha}^{\epsilon, \delta}\right|^{2} d x d t\right)^{\frac{1}{2}}
\end{aligned}
$$

Using that for $a, b \in \mathbb{R}$, one has $a b \leq m_{\mathbf{K}} \frac{a^{2}}{2}+\frac{b^{2}}{2 m_{\mathbf{K}}}$, we obtain the existence of $C$ depending only on $\mathbf{K}, \rho_{\alpha}, \mu_{\alpha}, \Omega, T$, $\mathbf{g}$ such that

$$
\iint_{Q_{T}} \frac{k_{\alpha}^{\delta}\left(s^{\delta, \epsilon}\right)}{\mu_{\alpha}} \mathbf{K}^{\delta} \nabla p_{\alpha}^{\delta, \epsilon} \cdot \rho_{\alpha} \mathbf{g} d x d t \leq \frac{m_{\mathbf{K}}}{2} \iint_{Q_{T}} \frac{k_{\alpha}^{\delta}\left(s^{\delta, \epsilon}\right)}{\mu_{\alpha}}\left|\nabla p_{\alpha}^{\epsilon, \delta}\right|^{2} d x d t+C
$$

The inequality (33) is a consequence of (36)-(39). Since the function $p_{w}^{\epsilon}$ (and thus $p_{o}^{\epsilon}$ ) is defined up to a function depending on time, one can choose this function so that (35) holds.

Lemma 2.2 There exists $C^{\epsilon}$ depending only on $\pi, \phi, \mathbf{K}, \rho_{\alpha}, \mu_{\alpha}, \Omega, T, \mathbf{g}, \alpha_{M}$ and $\epsilon$ (but not on

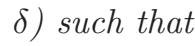

$$
\iint_{Q_{T}}\left(\nabla p_{\beta}^{\epsilon, \delta}\right)^{2} d x d t \leq C^{\epsilon}, \quad \text { for } \beta \in\{o, w\}
$$

Proof. We will prove this estimate only for the oil pressure, since obtaining it for the water pressure is similar.

$$
\begin{gathered}
\iint_{Q_{T}}\left(\nabla p_{o}^{\epsilon, \delta}\right)^{2} d x d t \leq \frac{1}{\alpha_{M}} \iint_{Q_{T}}\left(\frac{k_{o}^{\delta}\left(s^{\epsilon, \delta}\right)}{\mu_{o}}+\frac{k_{w}^{\delta}\left(s^{\epsilon, \delta}\right)}{\mu_{w}}\right)\left(\nabla p_{o}^{\epsilon, \delta}\right)^{2} d x d t \\
\quad \leq \frac{1}{\alpha_{M}} \iint_{Q_{T}}\left[\frac{k_{o}^{\delta}\left(s^{\epsilon, \delta}\right)}{\mu_{o}}\left(\nabla p_{o}^{\epsilon, \delta}\right)^{2}+\frac{k_{w}^{\delta}\left(s^{\epsilon, \delta}\right)}{\mu_{w}}\left(\nabla p_{w}^{\epsilon, \delta}+\nabla \pi^{\delta}\left(s^{\epsilon, \delta}\right)\right)^{2}\right] d x d t .
\end{gathered}
$$

Since $(a+b)^{2} \leq 2\left(a^{2}+b^{2}\right)$, and since $0 \leq k_{w}^{\delta}(s) \leq 1$, one obtains

$$
\begin{aligned}
& \iint_{Q_{T}}\left(\nabla p_{o}^{\epsilon, \delta}\right)^{2} d x d t \\
& \quad \leq \frac{1}{\alpha_{M}} \iint\left[\frac{k_{o}^{\delta}\left(s^{\epsilon, \delta}\right)}{\mu_{o}}\left(\nabla p_{o}^{\epsilon, \delta}\right)^{2}+2 \frac{k_{w}^{\delta}\left(s^{\epsilon, \delta}\right)}{\mu_{w}}\left(\nabla p_{w}^{\epsilon, \delta}\right)^{2}+\frac{1}{\mu_{w}}\left(\nabla \pi^{\delta}\left(s^{\epsilon, \delta}\right)\right)^{2}\right] d x d t .
\end{aligned}
$$


We conclude by using the energy estimate (33).

Let $h>0$, then we define $\Omega_{i}^{h}=\left\{x \in \Omega_{i}\right.$ s.t. $\operatorname{dist}\left(x, \Omega_{j}\right)>h$ for $\left.j \neq i\right\}$ and $Q_{i, T}^{h}=\Omega_{i}^{h} \times(0, T)$. On the set $\Omega_{i}^{\delta}$, the functions $f^{\delta}$ is equal to $f$ for all $f \in\left\{k_{\alpha}, \pi, \ldots\right\}$. This particularly yields that in $Q_{i, T}^{\delta}$, the two first equations of the system (32) can be rewritten under the form

$$
\begin{aligned}
& \phi_{i} \partial_{t} s^{\epsilon, \delta}-\operatorname{div}\left(\mathbf{K}_{i}\left(\frac{k_{o, i}\left(s^{\epsilon, \delta}\right)}{\mu_{o}}\left(\nabla P^{\epsilon, \delta}-\rho_{o} \mathbf{g}\right)+\nabla \varphi_{i}\left(s^{\epsilon, \delta}\right)\right)\right)=\epsilon \Delta \pi_{i}\left(s^{\epsilon, \delta}\right), \\
& -\operatorname{div}\left(\mathbf{K}_{i}\left(M_{i}\left(s^{\epsilon, \delta}\right) \nabla P^{\epsilon, \delta}-\zeta_{i}\left(s^{\epsilon, \delta}\right) \mathbf{g}\right)\right)=0 .
\end{aligned}
$$

Lemma 2.3 There exists $C$ depending only on $\phi, \mathbf{K}, \rho_{\alpha}, \mu_{\alpha}, \Omega, T, \mathbf{g}, \alpha_{M}$ (but neither on $\epsilon$ nor on $\delta$ ) such that for all $\epsilon, \delta>0$,

$$
\iint_{Q_{i, T}^{\delta}}\left(\nabla P^{\epsilon, \delta}\right)^{2} d x d t \leq C\left(1+\max _{i \in\{1,2\}}\left\|\pi_{i}\right\|_{L^{1}(0,1)}\right) .
$$

Proof. One has, thanks to the relation (19),

$$
\begin{aligned}
& \iint_{Q_{i, T}^{\delta}}\left(\nabla P^{\epsilon, \delta}\right)^{2} d x d t \leq \frac{1}{\alpha_{M}^{2}} \iint_{Q_{i, T}^{\delta}}\left(M_{i}\left(s^{\epsilon, \delta}\right) \nabla P^{\epsilon, \delta}\right)^{2} d x d t \\
& \quad \leq \frac{1}{\alpha_{M}^{2}} \iint_{Q_{i, T^{\delta}}}\left(\frac{k_{o, i}\left(s^{\epsilon, \delta}\right)}{\mu_{o}} \nabla p_{o}^{\epsilon, \delta}+\frac{k_{w, i}\left(s^{\epsilon, \delta}\right)}{\mu_{w}} \nabla p_{w}^{\epsilon, \delta}\right)^{2} d x d t \\
& \quad \leq \frac{2}{\min \left(\mu_{o}, \mu_{w}\right) \alpha_{M}^{2}} \iint_{Q_{i, T} \delta}\left(\frac{k_{o, i}\left(s^{\epsilon, \delta}\right)}{\mu_{o}}\left(\nabla p_{o}^{\epsilon, \delta}\right)^{2}+\frac{k_{w, i}\left(s^{\epsilon, \delta}\right)}{\mu_{w}}\left(\nabla p_{w}^{\epsilon, \delta}\right)^{2}\right) d x d t
\end{aligned}
$$

We conclude by using Proposition 2.1.

Lemma 2.4 There exists $C$ depending only on $\phi, \mathbf{K}, \rho_{\alpha}, \mu_{\alpha}, \Omega, T, \mathbf{g}, \alpha_{M}$ (but neither on $\epsilon$ nor on $\delta)$ such that for all $\epsilon, \delta>0$, one has:

$$
\iint_{Q_{i, T}^{\delta}}\left(\nabla \varphi_{i}\left(s^{\epsilon, \delta}\right)\right)^{2} d x d t \leq C\left(1+\max _{i \in\{1,2\}}\left\|\pi_{i}\right\|_{L^{1}(0,1)}\right) .
$$

Proof. This estimate is only a consequence of the fact that in $Q_{i, T}^{\delta}$,

$$
\nabla \varphi_{i}\left(s^{\epsilon, \delta}\right)=\frac{k_{o, i}\left(s^{\epsilon, \delta}\right)}{\mu_{o}}\left(\nabla p_{o}^{\epsilon, \delta}-\nabla P^{\epsilon, \delta}\right) .
$$

We conclude by using Proposition 2.1 and Lemma 2.3.

Lemma 2.5 Let $\tau \in(0, T)$ and let $h>0$, then there exists $C^{h}$ depending on $\phi, \mathbf{K}, \rho_{\alpha}, \mu_{\alpha}, \Omega, T$, g, $L_{\varphi_{i}}, \alpha_{M}$ and $h$ such that for all $\epsilon>0$ and for all $\delta \in(0, h)$,

$$
\iint_{Q_{i, T-\tau}^{2 h}}\left(\varphi_{i}\left(s^{\epsilon, \delta}\right)(\cdot, \cdot+\tau)-\varphi_{i}\left(s^{\epsilon, \delta}\right)\right)^{2} d x d t \leq \tau C^{h}\left(1+\max _{i \in\{1,2\}}\left\|\pi_{i}\right\|_{L^{1}(0,1)}\right) .
$$


Proof. Let $\xi^{h}$ be a nonnegative smooth function equal to 1 in $\Omega_{i}^{2 h}$ and equal to 0 in $\left(\Omega_{i}^{h}\right)^{c}$. Since $\varphi_{i}$ is Lipschitz continuous, one has

$$
\begin{aligned}
& \iint_{Q_{i, T-\tau}^{2 h}}\left(\varphi_{i}\left(s^{\epsilon, \delta}\right)(x, t+\tau)-\varphi_{i}\left(s^{\epsilon, \delta}\right)(x, t)\right)^{2} d x d t \\
& \leq \quad \iint_{Q_{i, T-\tau}^{h}} \xi^{h}(x)\left(\varphi_{i}\left(s^{\epsilon, \delta}\right)(x, t+\tau)-\varphi_{i}\left(s^{\epsilon, \delta}\right)(x, t)\right)^{2} d x d t \\
& \leq \quad L_{\varphi_{i}} \iint_{Q_{i, T-\tau}^{h}} \xi^{h}(x)\left(\varphi_{i}\left(s^{\epsilon, \delta}\right)(x, t+\tau)-\varphi_{i}\left(s^{\epsilon, \delta}\right)(x, t)\right)\left(s^{\epsilon, \delta}(x, t+\tau)-s^{\epsilon, \delta}(x, t)\right) d x d t \\
& \leq \quad-\frac{L_{\varphi_{i}}}{\phi_{i}} \iint_{Q_{i, T-\tau}^{h}}\left[\int_{0}^{\tau}\left(\mathbf{K}_{i} \frac{k_{o, i}\left(s^{\epsilon, \delta}\right)(x, t+\theta)}{\mu_{o}}\left(\nabla p_{o}^{\epsilon, \delta}(x, t+\theta)-\rho_{o} \mathbf{g}\right)\right] d x d t\right. \\
& \quad \leq 2 \tau \frac{L_{\varphi_{i}}}{\phi_{i}}\left\|\nabla\left(\xi_{h} \varphi_{i}\left(s^{\epsilon, \delta}\right)\right)\right\|_{L^{2}\left(Q_{i, T}^{h}\right)} \times\left[\frac{\left.\left.\left(T \mid s^{\epsilon, \delta}\right)(x, t+\theta)\right) d \theta\right]}{\mu_{o}} \rho_{o}\left|\mathbf{K}_{i} \mathbf{g}\right|+\right. \\
& \left.\quad\left(2 \iint_{Q_{i, T}^{h}}\left(\left(\mathbf{K}_{i} \frac{k_{o, i}\left(s^{\epsilon, \delta}\right)}{\mu_{o}} \nabla p_{o}^{\epsilon, \delta}\right)^{2}+\epsilon\left(\nabla \pi_{i}\left(s^{\epsilon, \delta}\right)\right)^{2}\right) d x d t\right)^{\frac{1}{2}}\right] .
\end{aligned}
$$

Moreover, since $\left\|\varphi_{i}\right\|_{\infty} \leq L_{\varphi_{i}}$, there exists $C^{h}$ depending only on $\Omega$ and $h$ such that

$$
\left\|\nabla\left(\xi_{h} \varphi_{i}\left(s^{\epsilon, \delta}\right)\right)\right\|_{L^{2}\left(Q_{i, T}^{h}\right)} \leq C^{h} L_{\varphi_{i}}+\sqrt{2}\left\|\nabla \varphi_{i}\left(s^{\epsilon, \delta}\right)\right\|_{L^{2}\left(Q_{i, T}^{h}\right)} .
$$

One concludes by using Proposition 2.1 and Lemma 2.4.

We have now all the necessary estimates to consider the limit $\delta \rightarrow 0$ of our problem.

Proposition 2.6 There exists $s^{\epsilon} \in L^{\infty}\left(Q_{T} ;[0,1]\right), p_{o}^{\epsilon}, p_{w}^{\epsilon} \in L^{2}\left((0, T) ; H^{1}(\Omega)\right)$ solution to the system (31). Moreover, there exists $C$ depending only on $\phi, \mathbf{K}, \rho_{\alpha}, \mu_{\alpha}, \Omega, T, \mathbf{g}, \alpha_{M}$ such that

$$
\begin{aligned}
& \frac{m_{\mathbf{K}}}{2} \sum_{\alpha \in\{o, w\}} \iint_{Q_{T}} \frac{k_{\alpha, i}\left(s^{\epsilon}\right)}{\mu_{\alpha}}\left(\nabla p_{\alpha}^{\epsilon}\right)^{2} d x d t \\
& \quad+\epsilon \iint_{Q_{T}}\left(\nabla \pi\left(s^{\epsilon}\right)\right)^{2} d x d t \leq C\left(1+\max _{i \in\{1,2\}}\left\|\pi_{i}\right\|_{L^{1}(0,1)}\right) .
\end{aligned}
$$

Furthermore, for $i \in\{1,2\}$, one has $P^{\epsilon}, \varphi_{i}\left(s^{\epsilon}\right) \in L^{2}\left((0, T) ; H^{1}\left(\Omega_{i}\right)\right)$ with

$$
P_{1}^{\epsilon}-\lambda_{w, 1}\left(\pi_{1}\left(s_{1}^{\epsilon}\right)\right)=P_{2}^{\epsilon}-\lambda_{w, 2}\left(\pi_{2}\left(s_{2}^{\epsilon}\right)\right), \quad \text { a.e. on } \Gamma \times(0, T),
$$

where $s_{i}^{\epsilon}$ denotes the trace on $\Gamma \times(0, T)$ from $Q_{i, T}$ of $s^{\epsilon}$. Moreover,

$$
\int_{\Omega} P^{\epsilon}(x, t) d x=0 \quad \text { for a.e. } t \in[0, T]
$$


and $\left(s^{\epsilon}, P^{\epsilon}\right)$ that satisfies the following system: $\forall \psi \in \mathcal{D}\left(\bar{\Omega}_{i} \times[0, T)\right)$,

$$
\begin{gathered}
\iint_{Q_{T}} \phi s^{\epsilon} \partial_{t} \psi d x d t+\int_{\Omega_{i}} \phi s_{0} \psi(\cdot, 0) d x \\
-\sum_{i \in\{1,2\}} \iint_{Q_{i, T}} \mathbf{K}_{i}\left(\frac{k_{o, i}\left(s^{\epsilon}\right)}{\mu_{o}}\left(\nabla P^{\epsilon}-\rho_{o} \mathbf{g}\right)+\nabla \varphi_{i}\left(s^{\epsilon}\right)+\epsilon \nabla \pi_{i}\left(s^{\epsilon}\right)\right) \cdot \nabla \psi d x d t=0 \\
\sum_{i \in\{1,2\}} \iint_{Q_{i, T}} \mathbf{K}_{i}\left(M_{i}\left(s^{\epsilon}\right) \nabla P^{\epsilon}-\zeta_{i}\left(s^{\epsilon}\right) \mathbf{g}\right) \cdot \nabla \psi d x d t=0 .
\end{gathered}
$$

The following energy estimate holds:

$$
\iint_{Q_{i, T}}\left(\nabla P^{\epsilon}\right)^{2} d x d t+\iint_{Q_{i, T}}\left(\nabla \varphi_{i}\left(s^{\epsilon}\right)\right)^{2} d x d t \leq C\left(1+\max _{i \in\{1,2\}}\left\|\pi_{i}\right\|_{L^{1}(0,1)}\right) .
$$

Let $h>0$ and $\tau \in(0, T)$, then there exists $C^{h}$ depending only on $\phi, \mathbf{K}, \rho_{\alpha}, \mu_{\alpha}, \Omega, T, \mathbf{g}, L_{\varphi_{i}}, \alpha_{M}$ and $h$ such that

$$
\iint_{Q_{i, T-\tau}^{h}}\left(\varphi_{i}\left(s^{\epsilon}\right)(\cdot, \cdot+\tau)-\varphi_{i}\left(s^{\epsilon}\right)\right)^{2} d x d t \leq \tau C^{h}\left(1+\max _{i \in\{1,2\}}\left\|\pi_{i}\right\|_{L^{1}(0,1)}\right) .
$$

Proof. Let $\epsilon$ be a fixed strictly positive parameter. First of all, since for all $\delta>0,0 \leq s^{\epsilon, \delta} \leq 1$ a.e. in $Q_{T}$, there exists $s^{\epsilon} \in L^{\infty}\left(Q_{T} ;[0,1]\right)$ such that, up to a subsequence,

$$
s^{\epsilon, \delta} \rightarrow s^{\epsilon} \quad \text { in the } L^{\infty}\left(Q_{T}\right) \text { weak- } \star \text { sense, and } 0 \leq s^{\epsilon} \leq 1 \text { a.e. in } Q_{T} .
$$

Let $h>0$. It follows from Lemma 2.4 that for all $\delta \in(0, h)$,

$$
\iint_{Q_{i, T}^{h}}\left(\nabla \varphi_{i}\left(s^{\epsilon, \delta}\right)\right)^{2} d x d t \leq C
$$

Then in particular, for all $\xi>0$, one has the following estimate on the space-translates of $\varphi_{i}\left(s^{\epsilon, \delta}\right)$ :

$$
\iint_{Q_{i, T}^{h+\xi}}\left(\varphi_{i}\left(s^{\epsilon, \delta}\right)(\cdot+\xi, \cdot)-\varphi_{i}\left(s^{\epsilon, \delta}\right)\right)^{2} d x d t \leq C \xi^{2}
$$

where $C$ does not depend on $\epsilon, \delta, \xi$ (see e.g. [Bré83]). Using moreover Lemma 2.5 allows to use the Kolmogorov compactness criterion (see e.g. [Bré83]) that provides that $\left(\varphi_{i}\left(s^{\epsilon, \delta}\right)\right)_{\delta \in(0, h)}$ is relatively compact in $L^{2}\left(Q_{i, T}^{h}\right)$. Hence, up to a subsequence, there exists a function $f \in L^{2}\left(Q_{i, T}^{h}\right)$ such that $\varphi_{i}\left(s^{\epsilon, \delta}\right)$ converges almost everywhere in $Q_{i, T}^{h}$ towards $f$. Since $\varphi_{i}^{-1}$ is continuous, one obtains that $s^{\epsilon, \delta}$ converges almost everywhere in $Q_{i, T}^{h}$ towards $\varphi_{i}^{-1}(f)=s^{\epsilon}$. Since this convergence results holds for all $h>0$, one obtains that, up to a subsequence,

$$
s^{\epsilon, \delta} \rightarrow s^{\epsilon} \quad \text { a.e. in } Q_{T} .
$$

Because of the definition (34) of the global pressure $P^{\epsilon, \delta}$ and thanks to (35), one has, for almost every $t \in[0, T]$ that

$$
\int_{\Omega} p_{w}^{\epsilon, \delta}(x, t) d x=-\int_{\Omega} \lambda_{w}^{\delta}\left(\pi^{\delta}\left(s^{\epsilon, \delta}\right)\right) d x .
$$


Hence, since we have supposed in this section that $\pi_{i} \in \mathcal{C}^{1}([0,1] ; \mathbb{R})$ and since $0 \leq\left(\lambda_{w}^{\delta}\right)^{\prime} \leq 1$, we obtain that

$$
\left|\int_{\Omega} p_{w}^{\epsilon, \delta}(x, t) d x\right| \leq\left\|\pi_{1}\right\|_{\infty}|\Omega| \text {. }
$$

Similarly, using the fact that $P^{\epsilon, \delta}=p_{o}^{\epsilon, \delta}+\lambda_{o}^{\delta}\left(\pi^{\delta}\left(s^{\epsilon, \delta}\right)\right)$ provides that for almost every $t \in[0, T]$, one has

$$
\left|\int_{\Omega} p_{o}^{\epsilon, \delta}(x, t) d x\right| \leq\left\|\pi_{1}\right\|_{\infty}|\Omega|
$$

Thanks to Lemma 2.2 and Poincaré-Wirtinger inequality, one can claim the existence of $C^{\epsilon}$ which is not depending on $\delta$ such that

$$
\left\|p_{\beta}^{\epsilon, \delta}-\frac{1}{|\Omega|} \int_{\Omega} p_{\beta}^{\epsilon, \delta}(x, \cdot) d x\right\|_{L^{2}\left((0, T) ; H^{1}(\Omega)\right)} \leq C^{\epsilon}, \quad \text { for } \beta \in\{o, w\} .
$$

This yields, using (53)-(54), that $\left(p_{\beta}^{\epsilon, \delta}\right)_{\delta}$ is uniformly bounded in $L^{2}\left((0, T) ; H^{1}(\Omega)\right)$. Thus there exits $p_{\beta}^{\epsilon}$ belonging to $L^{2}\left((0, T) ; H^{1}(\Omega)\right)$ such that, up to a subsequence,

$$
p_{\beta}^{\epsilon, \delta} \rightarrow p_{\beta}^{\epsilon} \quad \text { weakly in } L^{2}\left((0, T) ; H^{1}(\Omega)\right) .
$$

In particular, $\pi^{\delta}\left(s^{\epsilon, \delta}\right)=p_{o}^{\epsilon, \delta}-p_{w}^{\epsilon, \delta}$ also converges weakly in $L^{2}\left((0, T) ; H^{1}(\Omega)\right)$ and strongly in $L^{2}\left(Q_{T}\right)$ towards $\pi\left(s^{\epsilon}\right)$ thanks to (52). In order to check that $P^{\epsilon}$ satisfies the equation (45), it suffices to verify that $P^{\epsilon, \delta}$ tends weakly to $P^{\epsilon}$ in $L^{2}\left(Q_{T}\right)$. This convergence can be directly established using the definition (34) and (52)-(55).

Writing (32a) under a weak form and taking the no-flux boundary condition into account yield: $\forall \psi \in \mathcal{D}(\bar{\Omega} \times[0, T))$

$$
\begin{aligned}
& \iint_{Q_{T}} \phi^{\delta} s^{\epsilon, \delta} \partial_{t} \psi d x d t+\int_{\Omega} \phi^{\delta} s_{0} \psi(\cdot, 0) d x \\
& \quad-\iint_{Q_{T}} \mathbf{K}^{\delta} \frac{k_{o}^{\delta}\left(s^{\epsilon, \delta}\right)}{\mu_{o}}\left(\nabla p_{o}^{\epsilon, \delta}-\rho_{o} \mathbf{g}\right) \nabla \psi d x d t=\epsilon \iint_{Q_{T}} \nabla \pi^{\delta}\left(s^{\epsilon, \delta}\right) \nabla \psi d x d t
\end{aligned}
$$

while the weak formulation corresponding to (32b) is: $\forall \psi \in \mathcal{D}(\bar{\Omega} \times[0, T))$,

$$
\begin{aligned}
& \iint_{Q_{T}} \phi^{\delta} s^{\epsilon, \delta} \partial_{t} \psi d x d t+\int_{\Omega} \phi^{\delta} s_{0} \psi(\cdot, 0) d x \\
& \quad+\iint_{Q_{T}} \mathbf{K}^{\delta} \frac{k_{w}^{\delta}\left(s^{\epsilon, \delta}\right)}{\mu_{w}}\left(\nabla p_{w}^{\epsilon, \delta}-\rho_{w} \mathbf{g}\right) \nabla \psi d x d t=\epsilon \iint_{Q_{T}} \nabla \pi^{\delta}\left(s^{\epsilon, \delta}\right) \nabla \psi d x d t .
\end{aligned}
$$

Since $\phi^{\delta}$ and $\mathbf{K}^{\delta}$ converge almost everywhere respectively towards $\phi$ and $\mathbf{K}$, and since, thanks to $(52), k_{\beta}^{\delta}\left(s^{\epsilon, \delta}\right)$ tends almost everywhere - thus strongly in $L^{p}\left(Q_{T}\right)$ for all $p \in[1, \infty)$ - towards $k_{\beta}\left(s^{\epsilon}\right)$, one can pass to the limit in (56)-(57) using (52) and (55), obtaining

$$
\begin{aligned}
& \iint_{Q_{T}} \phi s^{\epsilon} \partial_{t} \psi d x d t+\int_{\Omega} \phi s_{0} \psi(\cdot, 0) d x \\
& \quad-\iint_{Q_{T}} \mathbf{K} \frac{k_{o}\left(s^{\epsilon}\right)}{\mu_{o}}\left(\nabla p_{o}^{\epsilon}-\rho_{o} \mathbf{g}\right) \nabla \psi d x d t=\epsilon \iint_{Q_{T}} \nabla \pi\left(s^{\epsilon}\right) \nabla \psi d x d t
\end{aligned}
$$


and

$$
\begin{aligned}
& \iint_{Q_{T}} \phi s^{\epsilon} \partial_{t} \psi d x d t+\int_{\Omega} \phi s_{0} \psi(\cdot, 0) d x \\
& \quad+\iint_{Q_{T}} \mathbf{K} \frac{k_{w}\left(s^{\epsilon}\right)}{\mu_{w}}\left(\nabla p_{w}^{\epsilon}-\rho_{w} \mathbf{g}\right) \nabla \psi d x d t=\epsilon \iint_{Q_{T}} \nabla \pi\left(s^{\epsilon}\right) \nabla \psi d x d t .
\end{aligned}
$$

Thanks to (52) and (55), one can also pass in the limit in the relation $p_{o}^{\epsilon, \delta}-p_{w}^{\epsilon, \delta}=\pi^{\delta}\left(s^{\epsilon, \delta}\right)$, leading to

$$
p_{o}^{\epsilon}-p_{w}^{\epsilon}=\pi\left(s^{\epsilon}\right)
$$

then $s^{\epsilon}, p_{o}^{\epsilon}$ and $p_{w}^{\epsilon}$ are solutions to (31).

Since in $Q_{i, T}$, the function $P^{\epsilon}$ has been built so that

$$
M_{i}\left(s^{\epsilon}\right) \nabla P^{\epsilon}=\frac{k_{o, i}\left(s^{\epsilon}\right)}{\mu_{o}} \nabla p_{o}^{\epsilon}+\frac{k_{w, i}\left(s^{\epsilon}\right)}{\mu_{w}} \nabla p_{w}^{\epsilon},
$$

classical calculations (see e.g. [AKM90], [CJ86]) yield that the weak formulation (58)-(59) is equivalent to (46)-(47).

Let $h>0$, then for all $\delta \in(0, h)$, one has

$$
\nabla P^{\epsilon, \delta}=\nabla p_{w}^{\epsilon, \delta}+\frac{k_{o, i}\left(s^{\epsilon, \delta}\right)}{k_{o, i}\left(s^{\epsilon, \delta}\right)+\frac{\mu_{o}}{\mu_{w}} k_{w, i}\left(s^{\epsilon, \delta}\right)} \nabla \pi_{i}\left(s^{\epsilon, \delta}\right) \quad \text { a.e. in } Q_{T}^{h} .
$$

Thus it follows from (52)-(55) that $\nabla P^{\epsilon, \delta}$ converges towards $\nabla P^{\epsilon}$ weakly in $L^{2}\left(Q_{T}^{h}\right)$ as $\delta$ tends to 0 . This ensures that

$$
\iint_{Q_{i, T}^{h}}\left(\nabla P^{\epsilon}\right)^{2} d x \leq \liminf _{\delta \rightarrow 0} \iint_{Q_{i, T}^{h}}\left(\nabla P^{\epsilon, \delta}\right)^{2} d x
$$

Thanks to Lemma 2.3, one obtains that for all $h>0$,

$$
\iint_{Q_{i, T}^{h}}\left(\nabla P^{\epsilon}\right)^{2} d x \leq C\left(1+\max _{i \in\{1,2\}}\left\|\pi_{i}\right\|_{L^{1}(0,1)}\right) .
$$

Letting now $h$ tend to 0 provides the estimate (48). The estimates (43) and (49) are directly provided by letting $\delta$ tend to 0 in the estimates (33) and (42).

Since $p_{w}^{\epsilon} \in L^{2}\left((0, T) ; H^{1}(\Omega)\right)$, then it is continuous on $\Gamma \times(0, T)$ in the sense that its traces from $Q_{1, T}$ and $Q_{2, T}$ coincide. Using the definition (15) of the global pressure provides (44).

\subsection{Proof of Theorem 2}

The goal of this section is to let tend $\epsilon$ to 0 in the system (31). We first give the following technical lemma, that ensures that the global pressure jump at the interface remains uniformly bounded, and that remains valid for non-matching capillary pressure functions.

Lemma 2.7 Let $\pi_{1}, \pi_{2}$ be increasing functions belonging to $\mathcal{C}^{1}((0,1) ; \mathbb{R}) \cap L^{1}((0,1))$, then the functions $\lambda_{w, i}$, defined by (16), are non-decreasing and bounded on $\mathbb{R}_{-}$while the functions $\lambda_{o, i}$, defined by (17), are non-increasing and bounded on $\mathbb{R}_{+}$. As a consequence, the function

$$
p \mapsto Z(p)=\lambda_{w, 1}(p)-\lambda_{w, 2}(p)=\lambda_{o, 1}(p)-\lambda_{o, 2}(p)
$$

is bounded on $\mathbb{R}$ by a quantity depending only on $k_{\alpha, i}, \mu_{\alpha}$ and $\left\|\pi_{i}\right\|_{L^{1}((0,1))}(i \in\{1,2\}, \alpha \in\{o, w\})$, and admits finite limits as $p \rightarrow \pm \infty$. 
Proof. Denote by $\mathcal{L}_{i}$ the Lipschitz constant of $s \mapsto \frac{k_{o, i}(s)}{k_{o, i}(s)+\frac{\mu_{o}}{\mu_{w}} k_{w, i}(s)}$, then, for all $p \leq 0$,

$$
\lambda_{w, i}(p)=\int_{0}^{p} \frac{k_{o, i}\left(\pi_{i}^{-1}(a)\right)}{k_{o, i}\left(\pi_{i}^{-1}(a)\right)+\frac{\mu_{o}}{\mu_{w}} k_{w, i}\left(\pi_{i}^{-1}(a)\right)} d a \geq-\mathcal{L}_{i} \int_{p}^{0}\left|\pi_{i}^{-1}(a)\right| d a .
$$

Then it follows from (7) that

$$
0 \geq \lambda_{w, i}(p) \geq-\mathcal{L}_{i}\left\|\pi_{i}^{-1}\right\|_{L^{1}\left(\mathbb{R}_{-}\right)} \geq-\mathcal{L}_{i}\left\|\pi_{i}\right\|_{L^{1}(0,1)}, \quad \forall p \leq 0,
$$

and thus that, for all $p \leq 0,|Z(p)| \leq \mathcal{L}_{i}\left\|\pi_{i}\right\|_{L^{1}(0,1)}$. In order to deal with the case $p \geq 0$, we remark that, thanks to (18), $Z(p)$ is also equal to $\lambda_{o, 1}(p)-\lambda_{o, 2}(p)$, where

$$
\lambda_{o, i}(p)=\int_{0}^{p}\left(\frac{k_{o, i}\left(\pi_{i}^{-1}(a)\right)}{k_{o, i}\left(\pi_{i}^{-1}(a)\right)+\frac{\mu_{o}}{\mu_{w}} k_{w, i}\left(\pi_{i}^{-1}(a)\right)}-1\right) d a \geq-\mathcal{L}_{i} \int_{0}^{p}\left|\pi_{i}^{-1}(a)-1\right| d a .
$$

Hence, for all $p \geq 0$,

$$
0 \geq \lambda_{o, i}(p) \geq-\mathcal{L}_{i}\left\|\pi_{i}^{-1}-1\right\|_{L^{1}\left(\mathbb{R}_{+}\right)} \geq-\mathcal{L}_{i}\left\|\pi_{i}\right\|_{L^{1}((0,1))} .
$$

This yields that for all $p \geq 0,|Z(p)| \leq \mathcal{L}_{i}\left\|\pi_{i}\right\|_{L^{1}((0,1))}$. The fact that $Z(p)$ admits a finite limit as $p \rightarrow-\infty$ comes from the fact that so does $\lambda_{w, i}(p)$, while $\lambda_{o, i}(p)$ admits a finite limit as $p \rightarrow+\infty$, ensuring that it is also the case for $Z(p)$.

Lemma 2.8 Denote by $m_{i}\left(P^{\epsilon}\right)(t)=\frac{1}{\Omega_{i} \mid} \int_{\Omega_{i}} P^{\epsilon}(x, t) d x$, then there exists $C$ depending only on $k_{\alpha, j}$, $\left\|\pi_{j}\right\|_{L^{1}((0,1))}, \phi, \mathbf{K}, \rho_{\alpha}, \mu_{\alpha}, \Omega, T, \mathbf{g}(j \in\{1,2\}, \alpha \in\{o, w\})$ such that

$$
\left\|m_{i}\left(P^{\epsilon}\right)\right\|_{L^{2}((0, T))} \leq C .
$$

Proof. It follows from (45) that for almost all $t \in(0, T)$, one has

$$
\left|\Omega_{1}\right| m_{1}\left(P^{\epsilon}\right)(t)+\left|\Omega_{2}\right| m_{2}\left(P^{\epsilon}\right)(t)=0 .
$$

Thanks to (44), the following relation holds almost everywhere on $\Gamma \times(0, T)$ :

$$
m_{1}\left(P^{\epsilon}\right)-m_{2}\left(P^{\epsilon}\right)=\left(P_{2}^{\epsilon}-m_{2}\left(P^{\epsilon}\right)\right)-\left(P_{1}^{\epsilon}-m_{1}\left(P^{\epsilon}\right)\right)-\lambda_{w, 2}\left(\pi_{2}\left(s_{2}^{\epsilon}\right)\right)+\lambda_{w, 1}\left(\pi_{1}\left(s_{1}^{\epsilon}\right)\right),
$$

ensuring, thanks to (60), that

$$
\left(m_{1}\left(P^{\epsilon}\right)\right)^{2} \leq C\left(\left(P_{2}^{\epsilon}-m_{2}\left(P^{\epsilon}\right)\right)^{2}+\left(P_{1}^{\epsilon}-m_{1}\left(P^{\epsilon}\right)\right)^{2}+\left(\lambda_{w, 2}\left(\pi_{2}\left(s_{2}^{\epsilon}\right)\right)-\lambda_{w, 1}\left(\pi_{1}\left(s_{1}^{\epsilon}\right)\right)\right)^{2}\right) .
$$

Integrating this relation on $\Gamma \times(0, T)$ provides

$$
|\Gamma| \int_{0}^{T}\left(m_{1}\left(P^{\epsilon}\right)(t)\right)^{2} d t \leq A_{2}^{\epsilon}+A_{1}^{\epsilon}+B^{\epsilon},
$$

where, using that $\pi_{1}\left(s_{1}^{\epsilon}\right)=\pi_{2}\left(s_{2}^{\epsilon}\right)$, we have set

$$
\begin{aligned}
A_{i}^{\epsilon} & =C \int_{0}^{T} \int_{\Gamma}\left(P_{i}^{\epsilon}-m_{i}\left(P^{\epsilon}\right)\right)^{2} d x d t \\
B^{\epsilon} & =C \int_{0}^{T} \int_{\Gamma} Z\left(\pi_{i}\left(s_{i}^{\epsilon}\right)\right)^{2} d x d t
\end{aligned}
$$


where the function $Z$ was introduced in Lemma 2.7. Thanks to Lemma 2.7, there exists $C$ depending only on the prescribed data such that

$$
B^{\epsilon} \leq C
$$

Thanks to the continuity of the trace operator mapping $H^{1}\left(\Omega_{i}\right)$ to $L^{2}(\Gamma)$, there exists $C$ depending only on $\Omega_{i}$ such that

$$
\left\|P_{i}^{\epsilon}-m_{i}\left(P^{\epsilon}\right)\right\|_{L^{2}(\Gamma \times(0, T))} \leq C\left\|P^{\epsilon}-m_{i}\left(P^{\epsilon}\right)\right\|_{L^{2}\left((0, T) ; H^{1}\left(\Omega_{i}\right)\right)} .
$$

Now, from Poincaré-Wirtinger inequality and estimate (48), one has

$$
\left\|P^{\epsilon}-m_{i}\left(P^{\epsilon}\right)\right\|_{L^{2}\left((0, T) ; H^{1}\left(\Omega_{i}\right)\right)} \leq C\left\|\nabla P^{\epsilon}\right\|_{L^{2}\left(Q_{i, T}\right)} \leq C,
$$

where $C$ only depends on the prescribed data. As a consequence, there exists $C$ depending only on the prescribed data such that

$$
A_{i}^{\epsilon} \leq C
$$

It follows then from (61)-(63) that $\int_{0}^{T}\left(m_{1}\left(P^{\epsilon}\right)(t)\right)^{2} d t \leq C$. The derivation of an $L^{2}((0, T))$-estimate on $m_{2}\left(P^{\epsilon}\right)$ may then be deduced from (60).

We now give the following lemma, which is a straightforward consequence of (48), Lemma 2.8 and the Poincaré-Wirtinger inequality.

Lemma 2.9 There exists $P \in L^{2}\left((0, T) ; H^{1}\left(\Omega_{i}\right)\right)$ such that, up to a subsequence, $P^{\epsilon}$ converges towards $P$ weakly in $L^{2}\left((0, T) ; H^{1}\left(\Omega_{i}\right)\right)$ as $\epsilon$ tends to 0 . Moreover,

$$
\|P\|_{L^{2}\left((0, T) ; H^{1}\left(\Omega_{i}\right)\right)} \leq C,
$$

where $C$ only depends on $k_{\alpha, j},\left\|\pi_{j}\right\|_{L^{1}((0,1))}, \phi, \mathbf{K}, \rho_{\alpha}, \mu_{\alpha}, \Omega, T, \mathbf{g}(j \in\{1,2\}, \alpha \in\{o, w\})$.

Lemma 2.10 There exists $s \in L^{\infty}\left(Q_{T} ;[0,1]\right)$ such that, up to a subsequence,

$$
\begin{aligned}
& s^{\epsilon} \rightarrow s \text { a.e. in } Q_{T} \text { as } \epsilon \rightarrow 0, \\
& \varphi_{i}\left(s^{\epsilon}\right) \rightarrow \varphi_{i}(s) \text { weakly in } L^{2}\left((0, T) ; H^{1}\left(\Omega_{i}\right)\right) .
\end{aligned}
$$

Moreover, there exists $C$ depending only on $k_{\alpha, j},\left\|\pi_{j}\right\|_{L^{1}((0,1))}, \phi, \mathbf{K}, \rho_{\alpha}, \mu_{\alpha}, \Omega, T, \mathbf{g}(j \in\{1,2\}$, $\alpha \in\{o, w\})$ and $C^{h}$ depending only on $k_{\alpha, j},\left\|\pi_{j}\right\|_{L^{1}((0,1))}, \phi, \mathbf{K}, \rho_{\alpha}, \mu_{\alpha}, \Omega, T, \mathbf{g}(j \in\{1,2\}$, $\alpha \in\{o, w\}), L_{\varphi_{i}}$ and $h$ such that

$$
\begin{aligned}
& \left\|\varphi_{i}(s)\right\|_{L^{2}\left((0, T) ; H^{1}\left(\Omega_{i}\right)\right)} \leq C, \\
& \iint_{Q_{i, T-\tau}^{h}}\left(\varphi_{i}(s)(\cdot, \cdot+\tau)-\varphi_{i}(s)\right)^{2} d x d t \leq \tau C^{h},
\end{aligned}
$$

Proof. It follows from (48) and (49) that the family $\left(\varphi_{i}\left(s^{\epsilon}\right)\right)_{\epsilon}$ is sequentially relatively compact in $L^{2}\left(Q_{i, T}^{h}\right)$ for all $h>0$. Then there exists $f_{i} \in L^{2}\left(Q_{i, T}^{h}\right)$ such that, up to a subsequence,

$$
\varphi_{i}\left(s^{\epsilon}\right) \rightarrow f_{i} \text { a.e. in } Q_{i, T}^{h} \text { as } \epsilon \rightarrow 0 .
$$

Since this relation stands for all $h>0$, one can claim that

$$
\varphi_{i}\left(s^{\epsilon}\right) \rightarrow f_{i} \text { a.e. in } Q_{i, T} \text { as } \epsilon \rightarrow 0 .
$$


Using the fact that $\varphi_{i}^{-1}$ is continuous, then, by setting $s=\varphi_{i}^{-1}\left(f_{i}\right)$ in $Q_{i, T}$, we obtain that

$$
s^{\epsilon} \rightarrow s \text { a.e. in } Q_{T} \text { as } \epsilon \rightarrow 0 \text {. }
$$

Since $0 \leq s^{\epsilon} \leq 1$ almost everywhere in $Q_{T}$, we obtain that $s \in L^{\infty}\left(Q_{T} ;[0,1]\right)$. It follows from (48) that $\varphi_{i}\left(s^{\epsilon}\right)$ converges, up to a subsequence, towards $\varphi_{i}(s)$ weakly in $L^{2}\left((0, T) ; H^{1}\left(\Omega_{i}\right)\right)$, and that the estimate (64) holds for the limit, while the estimate (65) is obtained by passing to the limit in (49).

Lemma 2.11 The function $(x, t) \mapsto \epsilon \pi\left(s^{\epsilon}(x, t), x\right)$ tends to 0 in $L^{2}\left((0, T) ; H^{1}(\Omega)\right)$ as $\epsilon$ tends to 0 .

Proof. We deduce from the estimate (43) that

$$
\left\|\epsilon \pi\left(s^{\epsilon}, \cdot\right)\right\|_{L^{2}\left((0, T) ; H^{1}(\Omega)\right)} \leq C \epsilon^{1 / 2}
$$

ensuring the expected convergence.

We now state a proposition that ends the proof of Theorem 2 .

Proposition 2.12 Let $s, P$ be the functions built in Lemmas 2.9 and 2.10. Then $(s, P)$ is a solution to the problem (12)-(14), (20), (21), (23), (24), (29) in the sense of Definition 1.1.

Proof. The convergence properties stated in Lemmas 2.9, 2.10 and 2.11 allow to pass to the limit $\epsilon \rightarrow 0$ in the weak formulations (46) and (47). Since $P^{\epsilon}$ converges weakly in $L^{2}\left((0, T) ; H^{1}\left(\Omega_{i}\right)\right)$ for $i \in\{1,2\}$, it also converges weakly in $L^{2}\left(Q_{T}\right)$. As a consequence, we deduce from (45) that for all $\psi \in L^{2}((0, T))$,

$$
0=\int_{0}^{T}\left(\int_{\Omega} P^{\epsilon}(x, t) d x\right) \psi(t) d t \underset{\epsilon \rightarrow 0}{\longrightarrow} \int_{0}^{T}\left(\int_{\Omega} P(x, t) d x\right) \psi(t) d t
$$

ensuring that for almost all $t \in(0, T), \int_{\Omega} P(x, t) d x=0$.

Since $\varphi_{i}\left(s^{\epsilon}\right)$ converges towards $\varphi_{i}(s)$ weakly in $L^{2}\left((0, T) ; H^{1}\left(\Omega_{i}\right)\right)$ and strongly in $L^{2}\left(Q_{i, T}\right)$, then it also converges strongly in $L^{2}\left((0, T) ; H^{s}\left(\Omega_{i}\right)\right)$ for $s \in(1 / 2,1)$. As a consequence, its trace on $\Gamma \times(0, T)$ converges strongly in $L^{2}(\Gamma \times(0, T))$. The continuity of $\varphi_{i}^{-1}$ ensures the convergence of the traces $s_{i}^{\epsilon}$ towards $s_{i}$ almost everywhere on $\Gamma \times(0, T)$ (up to a subsequence) and in $L^{p}(\Gamma \times(0, T)$ ) for all $p \in[1, \infty)$. Hence, we can pass to the limit in the relation

$$
\pi_{1}\left(s_{1}^{\epsilon}\right)=\pi_{2}\left(s_{2}^{\epsilon}\right) \text { a.e. on } \Gamma \times(0, T),
$$

that gives $\pi_{1}\left(s_{1}\right)=\pi_{2}\left(s_{2}\right)$ a.e. on $\Gamma \times(0, T)$. Since $P^{\epsilon}$ converges towards $P$ weakly in $L^{2}\left((0, T) ; H^{1}\left(\Omega_{i}\right)\right)$, then $P_{i}^{\epsilon}$ converges towards $P_{i}$ weakly in $L^{2}(\Gamma \times(0, T))$. We can pass to the limit in the relation

$$
P_{1}^{\epsilon}-\lambda_{w, 1}\left(\pi_{1}\left(s_{1}^{\epsilon}\right)\right)=P_{2}^{\epsilon}-\lambda_{w, 2}\left(\pi_{2}\left(s_{2}^{\epsilon}\right)\right),
$$

that takes sense in $L^{2}(\Gamma \times(0, T))$, and that provides (29). 


\section{Existence for non-matching capillary pressure curves}

In this section, we aim to prove the existence of a weak solution in the case where the capillary pressure curve do not satisfy the assumption (28). As it has been done in [BLS09, CGP09] in the case where the elliptic equation on the pressure can be removed, as for example in the onedimensional case, the main idea consists in approximating the capillary pressure graphs $\tilde{\pi}_{i}$ by regularized capillary pressure functions $\pi_{i, n}$ satisfying the matching conditions (28).

Let $\left(\pi_{i, n}\right)_{n>1} \subset \mathcal{C}^{1}([0,1] ; \mathbb{R})$ be a sequence of approximate capillary pressures satisfying the matching condition (28), i.e. $\pi_{1, n}(0)=\pi_{2, n}(0)$ and $\pi_{1, n}(1)=\pi_{2, n}(1)$, such that

$$
\pi_{i, n}=\pi_{i} \quad \text { on }\left[\frac{1}{n}, 1-\frac{1}{n}\right]
$$

such that

$$
\pi_{i, n} \rightarrow \pi_{i} \text { in } L^{1}(0,1) \text { as } n \rightarrow \infty,
$$

and such that

$$
\pi_{i, n}(0) \rightarrow \min _{j} \pi_{j}(0), \quad \pi_{i, n}(1) \rightarrow \max _{j} \pi_{j}(1) \quad \text { as } n \rightarrow \infty,
$$

the quantities $\min _{j} \pi_{j}(0)$ and $\max _{j} \pi_{j}(0)$ belonging to $\overline{\mathbb{R}}$. In particular, due to (67), there exists $C_{\pi}$ not depending on $n$ fulfilling

$$
\int_{0}^{1}\left|\pi_{i, n}(a)\right| d a \leq C_{\pi}, \quad \forall i \in\{1,2\}, \forall n \geq 1,
$$

Note that Dini's theorem implies that

$$
\pi_{i, n}^{-1} \rightarrow \pi_{i}^{-1} \text { uniformly on } \mathbb{R} \text { as } n \rightarrow \infty .
$$

and that thanks to (67), one has furthermore that

$$
\left\|\pi_{i, n}^{-1}-\pi_{i}^{-1}\right\|_{L^{1}(\mathbb{R})} \rightarrow 0 \quad \text { as } n \rightarrow \infty .
$$

Remark 5. For proving (70), we use the following classical version of Dini's theorem: let $\left(f_{n}\right)_{n}$ be a sequence of continuous nondecreasing functions from $[0, \infty)$ into some bounded interval $[a, b] \subset R$ which converges pointwise to some continuous function and such that $\lim _{n \rightarrow \infty} f_{n}(\infty)=f(\infty)$, then, the convergence is uniform on $[0, \infty)$.

Denoting by

$$
\varphi_{i, n}(s)=\int_{0}^{s} \frac{k_{o, i}(a) k_{w, i}(a)}{\mu_{w} k_{o, i}(a)+\mu_{o} k_{w, i}(a)} \pi_{i, n}^{\prime}(a) d a,
$$

we assume furthermore that the sequence $\left(\pi_{i, n}\right)$ is chosen such that there exists $C_{\varphi}$, not depending on $n$, such that, for all $n \geq 1$,

$$
L_{\varphi_{i, n}}:=\left\|\varphi_{i, n}^{\prime}\right\|_{L^{\infty}(0,1)}=\left\|\frac{k_{o, i} k_{w, i}}{\mu_{w} k_{o, i}+\mu_{o} k_{w, i}} \pi_{i, n}^{\prime}\right\|_{L^{\infty}(0,1)} \leq C_{\varphi} .
$$




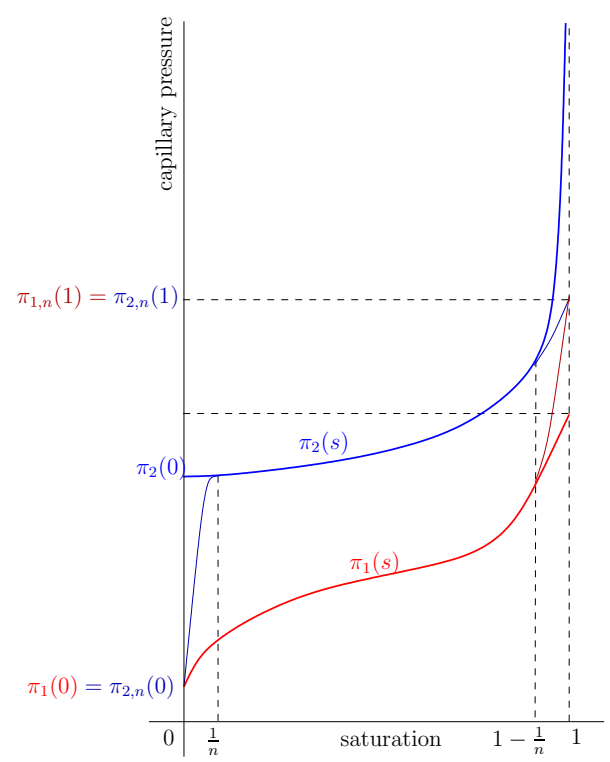

Figure 2: An example of functions $\pi_{i, n}$ fulfilling the matching condition (28) approximating non-matching capillary pressure functions $\pi_{i}$.

This particularly yields that $\varphi_{i, n} \rightarrow \varphi_{i}$ uniformly on $[0,1]$ as $n \rightarrow \infty$. One denotes by $\lambda_{w, i, n}$ then function defined by

$$
\lambda_{w, i, n}(p)=\int_{0}^{p} \frac{k_{o, i}\left(\pi_{i, n}^{-1}(a)\right)}{\frac{\mu_{o}}{\mu_{w}} k_{w, i}\left(\pi_{i, n}^{-1}(a)\right)+k_{o, i}\left(\pi_{i, n}^{-1}(a)\right)} d a, \quad \forall p \in \overline{\mathbb{R}},
$$

and

$$
\lambda_{o, i, n}(p)=-\int_{0}^{p} \frac{k_{w, i}\left(\pi_{i, n}^{-1}(a)\right)}{\frac{\mu_{w}}{\mu_{o}} k_{o, i}\left(\pi_{i, n}^{-1}(a)\right)+k_{w, i}\left(\pi_{i, n}^{-1}(a)\right)} d a, \quad \forall p \in \overline{\mathbb{R}} .
$$

Denoting by $\mathcal{L}_{i}$ a Lispchitz constant of $\frac{k_{o, i}}{\mu_{w} k_{w, i}+k_{o, i}}$, then, for all $p \in \mathbb{R}$, one has

$$
\begin{aligned}
\left|\lambda_{w, i, n}(p)-\lambda_{w, i}(p)\right| & \leq \mathcal{L}_{i} \int_{\min (0, p)}^{\max (0, p)}\left|\pi_{i, n}^{-1}(a)-\pi_{i}^{-1}(a)\right| d a \\
& \leq \mathcal{L}_{i}\left\|\pi_{i, n}^{-1}-\pi_{i}^{-1}\right\|_{L^{1}(\mathbb{R})} \rightarrow 0 \text { as } n \rightarrow \infty
\end{aligned}
$$

thanks to (71), so that

$$
\lambda_{w, i, n} \rightarrow \lambda_{w, i} \text { uniformly on } \mathbb{R} \text { as } n \rightarrow \infty .
$$

For any fixed $n$, Theorem 2 ensures the existence of a weak solution $\left(s_{n}, P_{n}\right)$ to the following approximate problem. In $Q_{i, T}$, we prescribe the conservations laws

$$
\begin{aligned}
& \phi_{i} \partial_{t} s_{n}-\operatorname{div}\left(\mathbf{K}_{i}\left(\frac{k_{o, i}\left(s_{n}\right)}{\mu_{o}}\left(\nabla P_{n}-\rho_{o} \mathbf{g}\right)+\nabla \varphi_{i, n}\left(s_{n}\right)\right)\right)=0, \\
& -\operatorname{div}\left(\mathbf{K}_{i}\left(M_{i}\left(s_{n}\right) \nabla P_{n}-\zeta_{i}\left(s_{n}\right) \mathbf{g}\right)\right)=0 .
\end{aligned}
$$


On $\Gamma \times(0, T)$, the transmission conditions are given by

$$
\begin{aligned}
& \pi_{1, n}\left(s_{1, n}\right)=\pi_{2, n}\left(s_{2, n}\right) \text { on } \Gamma \times(0, T), \\
& P_{1, n}-\lambda_{w, 1, n}\left(\pi_{1, n}\left(s_{1, n}\right)\right)=P_{2, n}-\lambda_{w, 2, n}\left(\pi_{2, n}\left(s_{2, n}\right)\right), \\
& \sum_{i \in\{1,2\}} \mathbf{K}_{i}\left(\frac{k_{o, i}\left(s_{n}\right)}{\mu_{o}}\left(\nabla P_{n}-\rho_{o} \mathbf{g}\right)+\nabla \varphi_{i, n}\left(s_{n}\right)\right) \cdot \mathbf{n}_{i}=0, \\
& \sum_{i \in\{1,2\}} \mathbf{K}_{i}\left(M_{i}\left(s_{n}\right) \nabla P_{n}-\zeta_{i}\left(s_{n}\right) \mathbf{g}\right) \cdot \mathbf{n}_{i}=0,
\end{aligned}
$$

where $s_{i, n}$ and $P_{i, n}$ denote the respective traces of $\left(s_{n}\right)_{\left.\right|_{\Omega_{i}}}$ and $\left(P_{n}\right)_{\left.\right|_{\Omega_{i}}}$ on $\Gamma \times(0, T)$. The system is closed by no-flux boundary conditions on $\partial \Omega \times(0, T)$ and the initial condition $s_{n}(\cdot, 0)=s_{0}$ in $\Omega$.

\subsection{Uniform estimates with respect to $n$ and compactness properties}

First of all, since for all $n \geq 1$, one has $s_{n} \in L^{\infty}\left(Q_{T} ;[0,1]\right)$, there exists $s \in L^{\infty}\left(Q_{T} ;[0,1]\right)$ such that, up to a subsequence,

$$
s_{n} \rightarrow s \text { in the } L^{\infty}\left(Q_{T}\right) \text { weak- } \star \text { sense. }
$$

Thanks to Lemma 2.10 and to the assumption (69) on the sequences $\left(\pi_{i, n}\right)_{n}$, there exists $C$ (not depending on $n$ ) such that

$$
\iint_{Q_{i, T}}\left(\nabla \varphi_{i, n}\left(s_{n}\right)\right)^{2} d x d t \leq C .
$$

Moreover, since $\left(\varphi_{i, n}\right)_{n}$ converges uniformly towards $\varphi_{i}$ on $[0,1]$, then $\left(\varphi_{i, n}\left(s_{n}\right)\right)_{n}$ is uniformly bounded in $L^{\infty}\left(Q_{T}\right) \subset L^{2}\left(Q_{T}\right)$. Thus there exists $f_{i} \in L^{2}\left((0, T) ; H^{1}\left(\Omega_{i}\right)\right)$ such that, up to a subsequence,

$$
\varphi_{i, n}\left(s_{n}\right) \rightarrow f_{i} \quad \text { weakly in } L^{2}\left((0, T) ; H^{1}\left(\Omega_{i}\right)\right) \text { as } n \rightarrow \infty .
$$

Let $\tau, h>0$, then it follows from Lemma 2.10 and (69) that there exists $C^{h}$ (not depending on $n$ ) such that

$$
\iint_{Q_{i, T-\tau}^{h}}\left(\varphi_{i, n}\left(s_{n}(\cdot, \cdot+\tau)\right)-\varphi_{i, n}\left(s_{n}\right)\right)^{2} d x d t \leq \tau C^{h} .
$$

Hence the sequence $\left(\varphi_{i, n}\left(s_{n}\right)\right)_{n}$ is relatively compact in $L^{2}\left(Q_{i, T}^{h}\right)$ for all $h>0$, thus also in $L^{2}\left(Q_{i, T}\right)$. Using Minty's trick (see e.g. [CGP09]), we obtain that up to a subsequence,

$$
\varphi_{i, n}\left(s_{n}\right) \rightarrow \varphi_{i}(s)=f_{i} \text { a.e. in } Q_{i, T} .
$$

Since $\varphi_{i}^{-1}$ is continuous, and since $\varphi_{i, n}$ converges uniformly to $\varphi_{i}$ (so that an adequate extension of $\varphi_{i}^{-1} \circ \varphi_{i, n}$ converges uniformly to the identity), we deduce that

$$
s_{n} \rightarrow s \quad \text { a.e. in } Q_{T} \text {. }
$$

Thanks to Lemma 2.9 and (69), we can claim that there exists $P \in L^{2}\left((0, T) ; H^{1}\left(\Omega_{i}\right)\right)$ such that

$$
P_{n} \rightarrow P \quad \text { weakly in } L^{2}\left((0, T) ; H^{1}\left(\Omega_{i}\right)\right) \text { as } n \rightarrow \infty .
$$


Thus $P_{n}$ tends to $P$ also weakly in $L^{2}\left(Q_{T}\right)$, hence, since $P_{n}$ satisfies

$$
\int_{\Omega} P_{n}(x, t) d x=0 \quad \text { for a.e. } t \in(0, T) .
$$

Using again arguments developed in the proof of Proposition 2.12, one obtains for $n$ tending to $\infty$ that

$$
\int_{\Omega} P(x, t) d x=0 \quad \text { for a.e. } t \in(0, T) .
$$

\subsection{Recovery of the weak formulations (25) and (26)}

Let $\psi \in \mathcal{C}_{c}^{\infty}(\bar{\Omega} \times[0, T))$, then thanks to (75), one has

$$
\iint_{Q_{T}} \phi s_{n} \partial_{t} \psi d x d t \rightarrow \iint_{Q_{T}} \phi s \partial_{t} \psi d x d t \quad \text { as } n \rightarrow \infty .
$$

Thanks to (76), (77) and (78), one has

$$
\begin{array}{r}
\lim _{n \rightarrow \infty} \sum_{i \in\{1,2\}} \iint_{Q_{i, T}} \mathbf{K}_{i}\left(\frac{k_{o, i}\left(s_{n}\right)}{\mu_{o}}\left(\nabla P_{n}-\rho_{o} \mathbf{g}\right)-\nabla \varphi_{i, n}\left(s_{n}\right)\right) \cdot \nabla \psi d x d t \\
=\sum_{i \in\{1,2\}} \iint_{Q_{i, T}} \mathbf{K}_{i}\left(\frac{k_{o, i}(s)}{\mu_{o}}\left(\nabla P-\rho_{o} \mathbf{g}\right)-\nabla \varphi_{i}(s)\right) \cdot \nabla \psi d x d t .
\end{array}
$$

The weak formulation (25) is then a direct consequence of (79) and (80).

The same way, in order to recover (26), it suffices to check that thanks to (77) and (78),

$$
\mathbf{K}_{i}\left(M_{i}\left(s_{n}\right) \nabla P_{n}-\zeta_{i}\left(s_{n}\right) \mathbf{g}\right) \rightarrow \mathbf{K}_{i}\left(M_{i}(s) \nabla P-\zeta_{i}(s) \mathbf{g}\right)
$$

weakly in $L^{2}\left(Q_{i, T}\right)$ as $n \rightarrow \infty$.

\subsection{Recovery of the transmission conditions on $\Gamma \times(0, T)$}

Since $P_{n}$ converges weakly towards $P$ in $L^{2}\left((0, T) ; H^{1}\left(\Omega_{i}\right)\right)$, one has

$$
P_{i, n} \rightarrow P_{i} \quad \text { weakly in } L^{2}(\Gamma \times(0, T)) \text { as } n \rightarrow \infty .
$$

Since the sequence $\left(\varphi_{i, n}\left(s_{n}\right)\right)$ converges (up to a subsequence) to $\varphi_{i}(s)$ weakly in $L^{2}\left((0, T) ; H^{1}\left(\Omega_{i}\right)\right)$ and strongly in $L^{2}\left(Q_{i, T}\right)$ and since $\Omega_{i}$ is supposed to be Lipschitz continuous, then for all $s \in\left(\frac{1}{2}, 1\right)$, the sequence $\left(\varphi_{i, n}\left(s_{n}\right)\right)$ converges strongly in $L^{2}\left((0, T) ; H^{s}\left(\Omega_{i}\right)\right)$. In particular, the trace $\varphi_{i, n}\left(s_{i, n}\right)$ converges strongly in $L^{2}(\Gamma \times(0, T))$ towards $\varphi_{i}\left(s_{i}\right)$, thus almost everywhere up to a new extraction. Applying again the arguments used to derive (77), we deduce that

$$
s_{i, n} \rightarrow s_{i} \text { a.e. on } \Gamma \times(0, T) .
$$

We denote by $\mathcal{U}$ and $\mathcal{V}$ the measurable sets of $\Gamma \times(0, T)$ defined by

$$
\mathcal{U}=\left\{(x, t) \in \Gamma \times(0, T) \mid\left\{s_{1}(x, t), s_{2}(x, t)\right\} \neq\{0,1\}\right\} \quad \text { and } \quad \mathcal{V}=\mathcal{U}^{c}
$$


It is worth noticing that $\mathcal{V}$ is negligible if $\min _{j} \pi_{j}(1)>\max _{j} \pi_{j}(0)$. Indeed, assume for instance that $s_{1}(x, t)=0, s_{2}(x, t)=1$; then, for $\alpha>0$ small and $n$ large enough, we have

$$
\pi_{2}(1-\alpha)=\pi_{2, n}(1-\alpha) \leq \pi_{2, n}\left(s_{2, n}\right)=\pi_{n}=\pi_{1, n}\left(s_{1, n}\right) \leq \pi_{1, n}(\alpha)=\pi_{1}(\alpha) .
$$

This implies $\pi_{2}(1-\alpha) \leq \pi_{1}(\alpha)$ and, letting $\alpha \rightarrow 0$ :

$$
\min _{j} \pi_{j}(1) \leq \pi_{2}(1) \leq \pi_{1}(0) \leq \max _{j} \pi_{j}(0) .
$$

We deduce the same in the case $s_{1}(x, t)=1, s_{2}(x, t)=0$.

In the sequel, we denote by $\mathcal{I}_{\pi}=\left[\min _{j} \pi_{j}(0), \max _{j} \pi_{j}(1)\right]$ and

$$
\pi_{n}:=\pi_{1, n}\left(s_{1, n}\right)=\pi_{2, n}\left(s_{2, n}\right) .
$$

Lemma 3.1 There exists a measurable function $\pi$ mapping $\mathcal{U}$ to $\mathcal{I}_{\pi}$ such that $\pi_{n}$ converges almost everywhere to $\pi$ on $\mathcal{U}$, and such that

$$
\pi \in \tilde{\pi}_{1}\left(s_{1}\right) \cap \tilde{\pi}_{2}\left(s_{2}\right) \quad \text { and } \quad P_{1}-\lambda_{w, 1}(\pi)=P_{2}-\lambda_{w, 2}(\pi) .
$$

Proof. Let $(x, t) \in \mathcal{U}$ such that $\left(s_{1, n}(x, t), s_{2, n}(x, t)\right)$ tends to $\left(s_{1}(x, t), s_{2}(x, t)\right)$.

- if $\left(s_{1}, s_{2}\right)=(0,0)$, then, for $\alpha>0$ and for $n$ large enough

$$
\pi_{1, n}(0)=\pi_{2, n}(0) \leq \pi_{n}=\pi_{1, n}\left(s_{1, n}\right)=\pi_{2, n}\left(s_{2, n}\right) \leq \min _{j} \pi_{j, n}(\alpha)=\min _{j} \pi_{j}(\alpha) .
$$

Since $\pi_{j, n}(0) \rightarrow \min _{j} \pi_{j}(0)$ as $n \rightarrow \infty$, it follows that $\pi_{n} \rightarrow \pi=\min _{j} \pi_{j}(0)$. In particular,

$$
\exists j \text { s.t. } \pi=\pi_{j}\left(s_{j}\right) \text { with } \pi \in \tilde{\pi}_{1}\left(s_{1}\right) \cap \tilde{\pi}_{2}\left(s_{2}\right) .
$$

Note that if one $\pi_{j}$ is unbounded near 0 , then $\min _{j} \pi_{j}(0)=-\infty=\pi \in \tilde{\pi}_{1}(0) \cap \tilde{\pi}_{2}(0)$.

- if $\left(s_{1}, s_{2}\right)=(1,1)$, we prove similarly that $\pi_{n} \rightarrow \pi=\max _{j} \pi_{j}(1)$ so that (85) remains valid.

- if we are not in the two previous cases, then, there exists $j$ such that $s_{j, n} \rightarrow s_{j} \in(0,1)$. In this case, for $n$ large enough, $\pi_{n}=\pi_{j, n}\left(s_{j, n}\right)=\pi_{j}\left(s_{j, n}\right)$ so that, by continuity, $\pi_{n} \rightarrow \pi_{j}\left(s_{j}\right)$. Assume for instance $j=1$. Then, either $s_{2} \in(0,1)$, in which case by continuity $\pi=\pi_{2}\left(s_{2}\right)$ also and (85) holds. If $s_{2}=0$, then since, for $\alpha>0$ small and $n$ large enough,

$$
\pi_{n}=\pi_{2, n}\left(s_{2, n}\right) \leq \pi_{2, n}(\alpha)=\pi_{2}(\alpha),
$$

we have $\pi \leq \pi_{2}(0)$. Therefore $\pi \in \tilde{\pi}_{2}(0)$ and again (85) holds. Finally, if $s_{2}=1$, we similarly have $\pi_{n}=\pi_{2, n}\left(s_{2, n}\right) \geq \pi_{2}(1-\alpha)$ and $\pi \geq \pi_{2}(1)$ or $\pi \in \tilde{\pi}_{2}(1)$ and (85) holds.

Now, let us pass to the limit in

$$
P_{1, n}-P_{2, n}=\lambda_{w, 1, n}\left(\pi_{n}\right)-\lambda_{w, 2, n}\left(\pi_{n}\right) .
$$

The left hand side converges weakly in $L^{2}(\Gamma \times(0, T))$ towards $P_{1}-P_{2}$, thus also weakly in $L^{2}(\mathcal{U})$. Thanks to Lemma 2.7, to the almost everywhere convergence on $\mathcal{U}$ of $\pi_{n}$ towards $\pi$, and to the 
uniform convergence $(73)$ of $\lambda_{w, i, n}$ towards $\lambda_{w, i}$, the righthand side converges strongly in $L^{2}(\mathcal{U})$ towards $\lambda_{w, 1}(\pi)-\lambda_{w, 2}(\pi)$. Then the relation

$$
P_{1}-P_{2}=\lambda_{w, 1}(\pi)-\lambda_{w, 2}(\pi)
$$

holds in $L^{2}(\mathcal{U})$, thus almost everywhere.

As it has been already noticed, the set $\mathcal{V}$ has to be taken in consideration only if

$$
\underline{\pi}:=\min _{i} \pi_{i}(1) \leq \bar{\pi}:=\max _{i} \pi_{i}(0) .
$$

Now, we assume that (86) is fulfilled. For any $\eta>0$, we denote by $T_{\eta}$ the function defined on $\mathbb{R}$ by

$$
T_{\eta}(c)=\min (\bar{\pi}+\eta, \max (\underline{\pi}-\eta, c))= \begin{cases}\frac{\pi}{c}-\eta & \text { if } c \leq \underline{\pi}-\eta, \\ c & \text { if } c \in[\underline{\pi}-\eta, \bar{\pi}+\eta], \\ \bar{\pi}+\eta & \text { if } c \geq \bar{\pi}+\eta .\end{cases}
$$

Lemma 3.2 There exists $\pi \in L^{\infty}(\mathcal{V} ;[\underline{\pi}, \bar{\pi}])$ such that, for all $\eta>0$, up to a subsequence,

$$
T_{\eta}\left(\pi_{n}\right) \rightarrow \pi \text { in the } L^{\infty}(\mathcal{V}) \text {-weak- } \star \text { sense as } n \rightarrow \infty .
$$

Proof. First, since the sequence $\left(T_{\eta}\left(\pi_{n}\right)\right)_{n}$ is uniformly bounded on $\mathcal{V}$, then there exists $\pi^{\eta} \in$ $L^{\infty}(\mathcal{V})$ such that, up to a subsequence,

$$
T_{\eta}\left(\pi_{n}\right) \rightarrow \pi^{\eta} \text { in the } L^{\infty}(\mathcal{V}) \text {-weak- } \star \text { sense as } n \rightarrow \infty .
$$

It remains to show that $\pi^{\eta}$ does not depend on $\eta$. Let $\eta_{1}, \eta_{2}>0$, then (up to a new subsequence), one has

$$
T_{\eta_{1}}\left(\pi_{n}\right)-T_{\eta_{2}}\left(\pi_{n}\right) \rightarrow \pi^{\eta_{1}}-\pi^{\eta_{2}} .
$$

Let $(x, t) \in \mathcal{V}$ such that $s_{1, n}(x, t) \rightarrow s_{1}(x, t)$ and $s_{2, n}(x, t) \rightarrow s_{2}(x, t)$, with $\left\{s_{1}(x, t), s_{2}(x, t)\right\}=$ $\{0,1\}$, then it follows from (84) that

$$
\liminf _{n} \pi_{n}(x, t) \geq \min _{i} \pi_{i}(1), \quad \limsup _{n} \pi_{n}(x, t) \leq \max _{i} \pi_{i}(0) .
$$

As a consequence,

$$
T_{\eta_{1}}\left(\pi_{n}(x, t)\right)-T_{\eta_{2}}\left(\pi_{n}(x, t)\right) \rightarrow 0 \text { a.e. }(x, t) \in \mathcal{V} \text { as } n \rightarrow \infty \text {. }
$$

By dominated convergence, this implies that $\left[T_{\eta_{1}}\left(\pi_{n}\right)-T_{\eta_{2}}\left(\pi_{n}\right)\right] \psi$ converges to 0 in $L^{1}(\mathcal{V})$ for all $\psi \in L^{1}(\mathcal{V})$ and, consequently, that $T_{\eta_{1}}\left(\pi_{n}\right)-T_{\eta_{2}}\left(\pi_{n}\right)$ converges also to 0 in $L^{\infty}(\mathcal{V})-$ weak $-\star$. We deduce $\pi^{\eta_{1}}=\pi^{\eta_{2}}$.

It remains to show that $\underline{\pi} \leq \pi(x, t) \leq \bar{\pi}$ for almost all $(x, t) \in \mathcal{V}$. Fix $\eta>0$, and assume, without loss of generality, that $\pi_{2}(1) \leq \pi_{1}(0)$. Using the fact that $T_{\eta}$ is nondecreasing, it follows from (83) that, for $n$ large enough,

$$
T_{\eta}\left(\pi_{2}(1-\alpha)\right) \leq T_{\eta}\left(\pi_{n}\right) \leq T_{\eta}\left(\pi_{1}(\alpha)\right) .
$$

Letting first $n$ tend to $\infty$, and then $\alpha$ tend to 0 gives

$$
T_{\eta}(\underline{\pi})=\underline{\pi} \leq \pi(x, t) \leq \bar{\pi}=T_{\eta}(\bar{\pi}) .
$$


Lemma 3.3 Let $\pi \in L^{\infty}(\mathcal{V})$ be the function defined by Lemma 3.2, then, for a.e. $(x, t) \in \mathcal{V}$, one has

$$
P_{1}(x, t)-P_{2}(x, t)=\lambda_{w, 1}(\pi(x, t))-\lambda_{w, 2}(\pi(x, t))
$$

Proof. First, thanks to (73), we can claim that

$$
\lambda_{w, 1, n}\left(\pi_{n}\right)-\lambda_{w, 2, n}\left(\pi_{n}\right)=\lambda_{w, 1}\left(\pi_{n}\right)-\lambda_{w, 2}\left(\pi_{n}\right)+\epsilon(n),
$$

with $\lim _{n \rightarrow \infty} \epsilon(n)=0$. Thus, since $P_{i, n} \rightarrow P_{i}$ weakly in $L^{2}(\mathcal{V})$, it is sufficient to show that

$$
\lambda_{w, 1}\left(\pi_{n}\right)-\lambda_{w, 2}\left(\pi_{n}\right) \rightarrow \lambda_{w, 1}(\pi)-\lambda_{w, 2}(\pi) \text { weakly in } L^{2}(\mathcal{V}) \text { as } n \rightarrow \infty .
$$

Let $\psi \in L^{2}(\mathcal{V})$, then, denoting by $Z(p)=\lambda_{w, 1}(p)-\lambda_{w, 2}(p)$, for all $\eta>0$,

$$
\iint_{\mathcal{V}} Z\left(\pi_{n}\right) \psi d x d t=A_{n}(\eta)+B_{n}(\eta)
$$

where one has

$$
A_{n}(\eta)=\iint_{\mathcal{V}} Z\left(T_{\eta}\left(\pi_{n}\right)\right) \psi d x d t, \quad B_{n}(\eta)=\iint_{\mathcal{V}}\left(Z\left(\pi_{n}\right)-Z\left(T_{\eta}\left(\pi_{n}\right)\right)\right) \psi d x d t .
$$

Fix $\epsilon>0$. Since, as stated in Lemma 2.7, $Z(p)$ admits finite limits as $p \rightarrow \pm \infty$, then there exists $R$ such that

$$
\eta>R \Longrightarrow\left\|Z-Z \circ T_{\eta}\right\|_{\infty} \leq \epsilon
$$

ensuring that

$$
\eta>R \Longrightarrow\left|B_{n}(\eta)\right| \leq C \epsilon .
$$

We suppose now, without loss of generality, that $\pi_{1}(1) \leq \pi_{2}(0)$. Then for almost all $(x, t) \in \mathcal{V}$, $s_{2}(x, t)=0$ and $s_{1}(x, t)=1$. One has

$$
\lambda_{w, 2}\left(T_{\eta}\left(\pi_{n}\right)\right)=\int_{0}^{\pi_{2}(0)} f_{2}(a) d a+\int_{\pi_{2}(0)}^{T_{\eta}\left(\pi_{n}\right)} f_{2}(a) d a
$$

where $f_{i}(p)=\frac{k_{o, i} \circ \pi_{i}^{-1}(p)}{\frac{\mu_{o}}{\mu_{w}} k_{w, i} \circ \pi_{2}^{-1}(p)+k_{o, i} \circ \pi_{i}^{-1}(p)}$. Note that $f_{i}(p)=0$ if $p \leq \pi_{i}(0)$, and $f_{i}(p)=1$ if $p \geq \pi_{i}(1)$. For almost all $(x, t) \in \mathcal{V}$, one has $\lim \sup _{n} \pi_{n}(x, t) \leq \pi_{2}(0)$, thus

$$
\lim _{n \rightarrow \infty} \lambda_{w, 2}\left(T_{\eta}\left(\pi_{n}(x, t)\right)\right)=\int_{0}^{\pi_{2}(0)} f_{2}(a) d a
$$

Similarly, the relation

$$
\lambda_{w, 1}\left(T_{\eta}\left(\pi_{n}\right)\right)-T_{\eta}\left(\pi_{n}\right)=\int_{0}^{\pi_{1}(1)}\left(f_{1}(a)-1\right) d a+\int_{\pi_{1}(1)}^{T_{\eta}\left(\pi_{n}\right)}\left(f_{1}(a)-1\right) d a
$$

yields that, for almost all $(x, t) \in \mathcal{V}$,

$$
\lim _{n \rightarrow \infty} \lambda_{w, 1}\left(T_{\eta}\left(\pi_{n}(x, t)\right)\right)-T_{\eta}\left(\pi_{n}\right)(x, t)=\int_{0}^{\pi_{1}(1)}\left(f_{1}(a)-1\right) d a .
$$


As a consequence of (89), (90) and Lemma 3.2, we obtain that, for all $\eta>0$,

$$
\lim _{n \rightarrow \infty} A_{n}(\eta)=C \iint_{\mathcal{V}} \psi d x d t+\iint \pi \psi d x d t,
$$

where

$$
C=\left(\int_{0}^{\pi_{1}(1)}\left[f_{1}(a)-1\right] d a-\int_{0}^{\pi_{2}(0)} f_{2}(a) d a\right) .
$$

Since $\pi_{1}(1)=\bar{\pi} \leq \pi \leq \underline{\pi}=\pi_{2}(0)$, we have

$$
\int_{\pi}^{\pi_{2}(0)} f_{2}(a) d a=0=\int_{\pi}^{\pi_{1}(1)}\left[f_{1}(a)-1\right] d a,
$$

so that

$$
C+\pi=\int_{0}^{\pi} f_{1}(a) d a-\int_{0}^{\pi} f_{2}(a) d a=\lambda_{w, 1}(\pi)-\lambda_{w, 2}(\pi),
$$

and

$$
\lim _{n \rightarrow \infty} A_{n}(\eta)=\iint_{\mathcal{V}} Z(\pi) \psi d x d t .
$$

In order to conclude the proof of Theorem 1, we gather the results of Lemmas 3.1, 3.2 and 3.3 in the following proposition.

Proposition 3.4 There exists a measurable function $\pi$ mapping $\Gamma \times(0, T)$ to $\overline{\mathbb{R}}$ such that, almost everywhere on $\Gamma \times(0, T)$, one has

$$
\pi \in \tilde{\pi}_{1}\left(s_{1}\right) \cap \tilde{\pi}_{2}\left(s_{2}\right) \quad \text { and } \quad P_{1}-\lambda_{w, 1}(\pi)=P_{2}-\lambda_{w, 2}(\pi) .
$$

\section{References}

[ABE96] B. Amaziane, A. Bourgeat, and H Elamri. Existence of solutions to various rock types model of two-phase flow in porous media. Applicable Analysis, 60:121-132, 1996.

[AC11] B. Andreianov and C. Cancès. Vanishing capillarity solutions of Buckley-Leverett equation with gravity in two-rocks' medium. HAL : hal-00631584 (submitted), 2011.

[AD85] H. W. Alt and E. DiBenedetto. Nonsteady flow of water and oil through inhomogeneous porous media. Ann. Scuola Norm. Sup. Pisa Cl. Sci. (4), 12(3):335-392, 1985.

[AKM90] S. N. Antontsev, A. V. Kazhikhov, and V. N. Monakhov. Boundary value problems in mechanics of nonhomogeneous fluids, volume 22 of Studies in Mathematics and its Applications. North-Holland Publishing Co., Amsterdam, 1990. Translated from the Russian.

[ALV84] H. W. Alt, S. Luckhaus, and A. Visintin. On nonstationary flow through porous media. Ann. Mat. Pura Appl. (4), 136:303-316, 1984.

[Arb92] T. Arbogast. The existence of weak solutions to single porosity and simple dualporosity models of two-phase incompressible flow. Nonlinear Anal., 19(11):1009-1031, 1992. 
[AS79] K. Aziz and A. Settari. Petroleum Reservoir Simulation. Elsevier Applied Science Publishers, Londres, 1979.

[BCH] K. Brenner, C. Cancès, and D. Hilhorst. Convergence of finite volume approximation for immiscible two-phase flows in porous media with discontinuous capillary pressure field in several dimensions. In preparation.

[BDPvD03] M. Bertsch, R. Dal Passo, and C. J. van Duijn. Analysis of oil trapping in porous media flow. SIAM J. Math. Anal., 35(1):245-267 (electronic), 2003.

[BH95a] A. Bourgeat and A. Hidani. Effective model of two-phase flow in a porous media made of different rock types. Applicable Analysis, 58:1-29, 1995.

[BH95b] A. Bourgeat and A. Hidani. A result of existence for a model of two-phase flow in a porous media made of different rock types. Applicable Analysis, 56:381-399, 1995.

[BLS09] F. Buzzi, M. Lenzinger, and B. Schweizer. Interface conditions for degenerate twophase flow equations in one space dimension. Analysis, 29:299-316, 2009.

[Bré83] H. Brézis. Analyse Fonctionnelle: Théorie et applications. Masson, 1983.

[Can08] C. Cancès. Nonlinear parabolic equations with spatial discontinuities. NoDEA Nonlinear Differential Equations Appl., 15(4-5):427-456, 2008.

[Can09] C. Cancès. Finite volume scheme for two-phase flow in heterogeneous porous media involving capillary pressure discontinuities. M2AN Math. Model. Numer. Anal., 43:973-1001, 2009.

[Can10a] C. Cancès. Asymptotic behavior of two-phase flows in heterogeneous porous media for capillarity depending only on space. I. Convergence to the optimal entropy solution. SIAM J. Math. Anal., 42(2):946-971, 2010.

[Can10b] C. Cancès. Asymptotic behavior of two-phase flows in heterogeneous porous media for capillarity depending only on space. II. Nonclassical shocks to model oil-trapping. SIAM J. Math. Anal., 42(2):972-995, 2010.

[Can10c] C. Cancès. On the effects of discontinuous capillarities for immiscible two-phase flows in porous media made of several rock-types. Netw. Heterog. Media, 5(3):635-647, 2010.

[Car99] J. Carrillo. Entropy solutions for nonlinear degenerate problems. Arch. Ration. Mech. Anal., 147(4):269-361, 1999.

[CG11] C. Cancès and T. Gallouët. On the time continuity of entropy solutions. J. Evol. Equ., 11(1):43-55, 2011.

[CGP09] C. Cancès, T. Gallouët, and A. Porretta. Two-phase flows involving capillary barriers in heterogeneous porous media. Interfaces Free Bound., 11(2):239-258, 2009.

[Che01] Z. Chen. Degenerate two-phase incompressible flow. I. Existence, uniqueness and regularity of a weak solution. J. Differential Equations, 171(2):203-232, 2001. 
[CJ86] G. Chavent and J. Jaffré. Mathematical Models and Finite Elements for Reservoir Simulation, volume 17. North-Holland, Amsterdam, stud. math. appl. edition, 1986.

[EEM06] G. Enchéry, R. Eymard, and A. Michel. Numerical approximation of a two-phase flow in a porous medium with discontinuous capillary forces. SIAM J. Numer. Anal., 43(6):2402-2422, 2006.

[EEN98] B.G. Ersland, M.S. Espedal, and R. Nybo. Numerical methods for flows in a porous medium with internal boundary. Comput. Geosci., 2:217-240, 1998.

[EMS09] A. Ern, I. Mozolevski, and L. Schuh. Discontinuous galerkin approximation of twophase flows in heterogeneous porous media with discontinuous capillary pressures. Submitted, 2009.

[Hid93] A. Hidani. Modlisation des coulements diphasiques en milieu poreux plusieurs types de roches. PhD thesis, Université de Saint-Etienne, 1993.

[Sch08] B. Schweizer. Homogenization of degenerate two-phase flow equations with oil trapping. SIAM J. Math. Anal., 39(6):1740-1763, 2008.

[vDEHP07] C. J. van Duijn, H. Eichel, R. Helmig, and I. S. Pop. Effective equations for two-phase flow in porous media: the effect of trapping on the microscale. Transp. Porous Media, 69(3):411-428, 2007.

[vDMdN95] C. J. van Duijn, J. Molenaar, and M. J. de Neef. The effect of capillary forces on immiscible two-phase flows in heterogeneous porous media. Transport in Porous Media, 21:71-93, 1995 .

[vDMP02] C. J. van Duijn, A. Mikelić, and I. S. Pop. Effective equations for two-phase flow with trapping on the micro scale. SIAM J. Appl. Math., 62:1531-1568, 2002. 